\title{
The Digital All-Pass Filter: A Versatile Signal Processing Building Block
}

PHILLIP A. REGALIA, STUdENT MEMBER, IEEE, SANJIT K. MITRA, FELlOW, IEeE, AND P. P. VAIDYANATHAN, MEMBER, IEEE

The digital all-pass filter is a computationally efficient signal processing building block which is quite useful in many signal processing applications. In this tutorial paper we review the properties of digital all-pass filters, and provide a broad overview of the diversity of applications in digital filtering. Starting with the definition and basic properties of a scalar all-pass function, a variety of structures satisfying the all-pass property are assembled, with emphasis placed on the concept of structural losslessness. Applications are then outlined in notch filtering, complementary filtering and filter banks, multirate filtering, spectrum and group-delay equalization, Hilbert transformations, and so on. In all cases, the structural losslessness property induces very robust performance in the face of multiplier coefficient quantization. Finally, the state-space manifestations of the all-pass property are explored, and it is shown that many all-pass filter structures are devoid of limit cycle behavior and feature very low roundoff noise gain.

\section{INTRODUCTION}

In many signal processing applications, the designer must determine the transfer function of a digital filter subject to constraints on the frequency selectivity and/or phase response which are dictated by the application at hand. Once a suitable transfer function is found, the designer must select a filter structure from the numerous choices available. Ultimately, finite precision arithmetic is used in any digital filter computation, and traditionally the roundoff noise and coefficient sensitivity characteristics have formed the basis of selecting one filter structure in favor of another.

In the quest for low coefficient sensitivity and low roundoff noise, an elegant theory of losslessness and passivity in the discrete-time domain has evolved [1], [2]. Although this theory has been motivated by the desire to obtain digital filters with predictable behavior under finite word-length conditions, many useful by-products have emerged which have contributed to a better understanding of computa-

Manuscript received July 10, 1987; revised October 16, 1987. This work was supported by the National Science Foundation under Grants MIP 85-08017 and MIP 84-04245.

P. A. Regalia and S. K. Mitra are with the Department of Electrical and Computer Engineering, University of California, Santa Barbara, CA 93106, USA.

P. P. Vaidyanathan is with the Department of Electrical Engineering, California Institute of Technology, Pasadena, CA 91125, USA.

IEEE Log Number $\mathbf{8 7 1 8 4 1 3 .}$ tionally efficient filter structures, tunable filters, filter bank analysis/synthesis systems, multirate filtering, and stability under linear and nonlinear (i.e., quantized) environments.

This paper considers a basic scalar lossless building block, which is a stable all-pass function. The interconnections of such lossless building blocks form useful solutions to many practical filtering problems. The many results presented here can be derived by appealing to elegant theoretical forms; however, in maintaining a tutorial tone it is our aim to expose the salient features using direct discrete-time concepts, in the hope that the references cited will further aid both the designer and researcher alike. We should point out that many of the results which are developed in terms of scalar all-pass functions in one dimension can be generalized to vector or matrix all-pass functions [3] and to multidimensional filtering [4], though for the present we restrict our attention to the one-dimensional scalar case.

We begin in Section II by defining a scalar all-pass function and reviewing some basic properties. Section III assembles a variety of all-pass filter structures, with emphasis placed on the concept of structural losslessness. Section IV outlines applications to notch filtering, Section V to complementary filters and filter banks, Section VI to multirate signal processing, Section VII to tunable filters, and Section VIII togroup-delay equalization. Finally, Section IX explores state-space representations of lossless transfer functions, and the implications of losslessness in obtaining very robust performance under finite word-length constraints.

\section{Definitions and Properties}

The frequency response $A\left(e^{j \omega}\right)$ of an all-pass filter exhibits unit magnitude at all frequencies, i.e.,

$$
\left|A\left(\mathrm{e}^{j \omega}\right)\right|^{2}=1, \quad \text { for all } \omega \text {. }
$$

The transfer function of such a filter has all poles and zeros occurring in conjugate reciprocal pairs, and takes the form

$$
A(z)=\mathrm{e}^{i \theta} \prod_{k=1}^{M} \frac{\gamma_{k}^{*}-z^{-1}}{1-\gamma_{k} z^{-1}} .
$$

For stability reasons we assume $\left|\gamma_{k}\right|<1$ for all $k$ to place all the poles inside the unit circle. Now, if $A(z)$ is constrained to be a real function, we must have $\theta=0$ or $\theta=\pi$, and any 
complex pole at $z=\gamma_{k}$ must be accompanied by a complex conjugate pole at $z=\gamma_{k}^{*}$. In this case $A(z)$ can be expressed in the form

$$
A(z)=\frac{z^{-M} D\left(z^{-1}\right)}{D(z)} .
$$

In effect, the numerator polynomial is obtained from the denominator polynomial by reversing the order of the coefficients. For example,

$$
A(z)=\frac{a_{2}+a_{1} z^{-1}+z^{-2}}{1+a_{1} z^{-1}+a_{2} z^{-2}}
$$

is a second-order all-pass function of the form of (2.3) above, since the numerator coefficients appear in the reverse order of those in the denominator. In this case, the numerator and denominator polynomials are said to form a mirror-image pair.

If we lift the restriction that $A(z)$ be a real function, then $A(z)$ takes the more general form

$$
A(z)=\frac{z^{-M} D^{*}\left(1 / z^{*}\right)}{D(z)}
$$

The numerator and denominator polynomials now form a Hermitian mirror-image pair. For example,

$$
\begin{aligned}
A(z) & =\frac{\alpha_{2}^{*}+\alpha_{1}^{*} z^{-1}+\alpha_{0}^{*} z^{-2}}{\alpha_{0}+\alpha_{1} z^{-1}+\alpha_{2} z^{-2}} \\
& =e^{j \theta} \frac{\left(\alpha_{2} / \alpha_{0}\right)^{*}+\left(\alpha_{1} / \alpha_{0}\right)^{*} z^{-1}+z^{-2}}{1+\left(\alpha_{1} / \alpha_{0}\right) z^{-1}+\left(\alpha_{2} / \alpha_{0}\right) z^{-2}}
\end{aligned}
$$

with $\theta=\arg \left(\alpha_{0}^{*} / \alpha_{0}\right)$, is recognized as a complex all-pass function due to the Hermitian mirror-image relation between the numerator and denominator polynomials.

\section{Properties}

From the definition of an all-pass function in (2.1), setting $A(z)=Y(z) / U(z)$ reveals

$$
\left|\gamma\left(e^{j \omega}\right)\right|^{2}=\left|U\left(e^{j \omega}\right)\right|^{2}, \quad \text { for all } \omega .
$$

Upon integrating both sides from $\omega=-\pi$ to $\pi$ and applying Parseval's relation [1], we obtain

$$
\sum_{n=-\infty}^{\infty}|y(n)|^{2}=\sum_{n=-\infty}^{\infty}|u(n)|^{2}
$$

It is convenient to interpret the two sides of (2.8) as the output energy and input energy of the digital filter, respectively [1], [5]. Thus an all-pass filter is lossless, since the output energy equals the input energy for all finite energy inputs. If the all-pass filter is stable as well, it is termed Lossless Bounded Real (LBR) [2], or more generally Lossless Bounded Complex [6] if the coefficients are not all real.

Another useful property follows from (2.1) with the aid of the maximum modulus theorem. In particular, since a stable all-pass function has all its poles inside the unit circle, all its zeros outside, and exhibits unit magnitude along the unit circle, one can deduce

$$
|A(z)| \begin{cases}<1, & \text { for }|z|>1 \\ =1, & \text { for }|z|=1 \\ >1, & \text { for }|z|<1\end{cases}
$$

This relation is useful in verifying stability in lattice realizations of all-pass filters.

The last property of interest is the change in phase for real all-pass filter over the frequency range $\omega \in[0, \pi]$. We start with the group delay function $\tau(\omega)$ of an all-pass filter, which is usually defined as

$$
\tau(\omega)=-\frac{d}{d \omega}\left[\arg A\left(e^{j \omega}\right)\right]
$$

Note that the phase function must be taken as continuous or "unwrapped" [5] if $\tau(\omega)$ is to be well-behaved. Since an all-pass function is devoid of zeros on the unit circle according to (2.1), the phase function arg $A\left(e^{j \omega}\right)$ can always be unwrapped with no ambiguities. Now, the phase response of a stable all-pass function is a monotonically decreasing function of $\omega$, so that $\tau(\omega)$ is everywhere positive. An $M$ thorder real all-pass function, in fact, satisfies the property

$$
\int_{0}^{\pi} \tau(\omega) d \omega=M \pi
$$

The interpretation of (2.11) is that the change in phase of the all-phase function as $\omega$ goes from 0 to $\pi$ is $-M \pi$ radians.

\section{All-Pass Filter Structures}

The (Hermitian) mirror-image symmetry relation between the numerator and denominator polynomials of an all-pass transfer function can be exploited to obtain a computationally efficient filter realization with a minimum number of multipliers. To see this, consider the second-order allpass function of (2.4) which, upon expressing $A(z)=$ $Y(z) / U(z)$, corresponds to the second-order difference equation

$$
\begin{aligned}
y(n)= & a_{2}[u(n)-y(n-2)] \\
& +a_{1}[u(n-1)-y(n-1)]+u(n-2)
\end{aligned}
$$

in which terms have been grouped in such a way that only two multiplications are required. A similar strategy can be applied to an arbitrary $M$ th-order all-pass filter, such that only $M$ multiplications are required to compute each output sample. On the other hand, a direct-form filter realization would in general require $2 M+1$ multiplications to compute each output sample. In this sense, an all-pass filter represents a computationally efficient structure.

The difference equation as expressed in (3.1) requires four delay (or storage) elements to be realized as a filter structure. Since the difference equation is of second order, this does not represent a canonic realization. However, minimum multiplier delay-canonic all-pass filter structures can be developed using the multiplier extraction approach [7], [8]. For example, consider the digital two-pair network of Fig. 1, which has a constraining multiplier $b_{1}$ at the second "port": $U_{2}(z)=b_{1} Y_{2}(z)$. The transfer function as seen from the remaining port is constrained to be a first-order all-pass

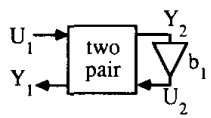

Fig. 1. Digital two-pair network constrained with a multiplier of value $b_{1}$. 
function

$$
\frac{y_{1}(z)}{U_{1}(z)}=\frac{z^{-1}-b_{1}}{1-b_{1} z^{-1}}
$$

This allows one to solve for the internal parameters of the digital two-pair. By following this and similar strategies, numerous first-order and second-order all-pass filter structures have been catalogued [7]-[10], many with roundoff noise expressions as functions of the pole locations, and with the minimum multiplier property. Such filter structures have the property that, upon quantizing the multiplier coefficients, the numerator polynomial and denominator polynomial retain a mirror-image relationship, and thus the all-pass characteristic is independent of any multiplier quantization. Filter structures with this property are termed structurally lossless or structurally LBR [2].

Another useful structure for realizing all-pass functions is the Gray and Markel lattice filter [11]. The synthesis procedure uses the following recursion [12]:

$$
\begin{aligned}
z^{-1} A_{m-1}(z)= & \frac{A_{m}(z)-k_{m}}{1-k_{m} A_{m}(z)^{\prime}} \\
& m=M, M-1, \cdots, 1
\end{aligned}
$$

where

$$
k_{m}=A_{m}(\infty)
$$

Now, with $A_{m}(z)$ a stable all-pass function, it can be verified using (2.10) that $\left|k_{m}\right|<1$, and that $A_{m-1}(\mathrm{z})$ is a stable all-pass function of one order lower. The structural interpretation of (3.3) for the first step in the recursion appears as Fig. 2,

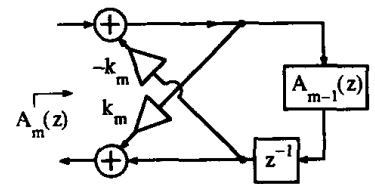

Fig. 2. Two multiplier lattice interpretation of all-pass function order reduction process.

where $A_{M-1}(z)$ is an $(M-1)$ th-order all-pass function. The recursion of (3.3) then continues on $A_{M-1}(z)$, and so on, which leads to the cascaded lattice realization of Fig. 3,

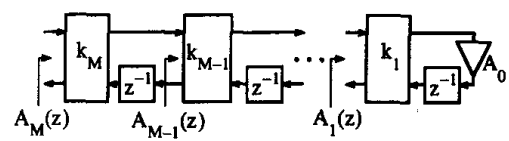

Fig. 3. The cascaded lattice implementation of the all-pass function $A_{M}(\mathrm{z})$.

where the constraining multiplier $A_{0}$ has unit magnitude. Each lattice two-pair is realized as in Fig. 2, although the overall transfer function is unaltered if each lattice two-pair is implemented as per Fig. 4(a) or (b). Fig. 4(a) is the single multiplier form [11], which requires the fewest number of multipliers. Fig. 4(b) is the normalized form [13], which has the advantage that all internal nodes are automatically scaled in the $\ell_{2}$ sense [14]. Although more multipliers are required, the four multiplications can be performed simultaneously using CORDIC processor techniques [15].

With all the lattice structures above, stability of the filter

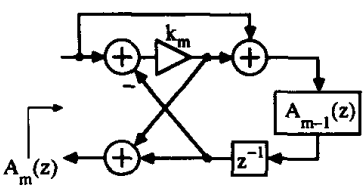

(a)

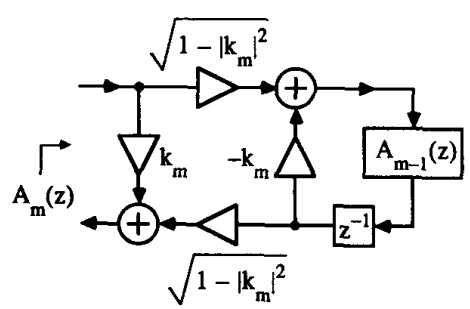

(b)

Fig. 4. (a) The single multiplier lattice two-pair. (b) The normalized lattice two-pair.

is equivalent to the condition that $\left|k_{m}\right|<1$ for all $m$. As such, the all-pass lattice filters form a useful model for checking stability of transfer functions; the connection of these lattice filters with system stability tests is explained in [12]. In addition, the mirror-image relation between the numerator and denominator polynomials holds in spite of coefficient quantization (except for the normalized form). Accordingly, the all-pass property for these lattice filters is structurally induced. These structures, in fact, have other favorable finite word-length properties which are explored in greater detail in Section IX.

The above discussion has assumed that $A_{M}(z)$ is a real allpass function, although the lattice filter is easily generalized for complex all-pass functions as well [6], [16]. In this case, the recursion of (3.3) becomes

$$
\begin{aligned}
z^{-1} A_{m-1}(z)= & \frac{A_{m}(z)-k_{m}^{*}}{1-k_{m} A_{m}(z)^{\prime}} \\
& m=M, M-1, \cdots, 1
\end{aligned}
$$

where now

$$
k_{m}=A_{m}^{*}(\infty) .
$$

The complex lattice two-pair now appears as Fig. 5. As in the real coefficient case, stability of $A_{M}(z)$ is equivalent to

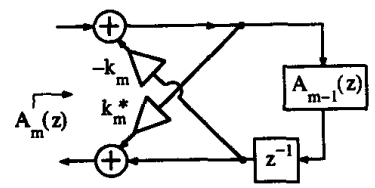

Fig. 5. Lattice filter interpretation of order reduction process for complex all-pass functions. The signals at all nodes are assumed to be complex-valued.

the condition $\left|k_{m}\right|<1$ for all $m$. The complex generalizations of the structures of Fig. 4 appear as Fig. 6, with properties similar to those of their real arithmetic counterparts.

An alternate method of realizing complex all-pass functions is to cascade first-order complex all-pass filters. Referring to (2.2), each term in the product corresponds to the 


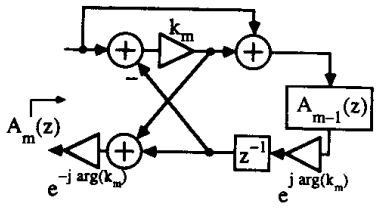

(a)

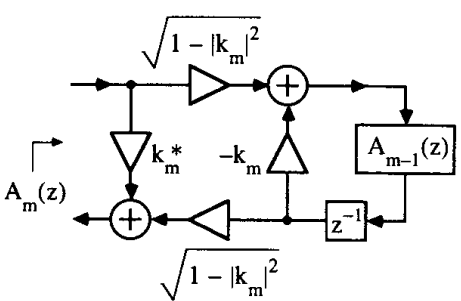

(b)

Fig. 6. Complex generalizations of the "single multiplier" lattice two-pair (a), and the normalized two-pair (b). The signals at all nodes are in general complex valued.

first-order difference equation

$$
y(n)=\gamma_{k} y(n-1)+\gamma_{k}^{*} u(n)-u(n-1)
$$

which may be arranged as

$$
\begin{aligned}
y(n)= & \operatorname{Re}\left\{\gamma_{k}\right\}[y(n-1)+u(n)] \\
& +j \operatorname{Im}\left\{\gamma_{k}\right\}[y(n-1)-u(n)]-u(n-1) .
\end{aligned}
$$

The corresponding realization is shown as Fig. 7, using four real multiplications (which is the equivalent of one complex

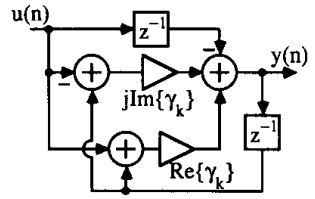

Fig. 7. Implementation of structurally lossless first-order complex all-pass filter. The signals at all nodes are in general complex-valued.

multiplication) and offering again the structurally induced all-pass property.

Finally, a complex all-pass function can be obtained from a real all-pass function using the frequency transformation [17], [18]

$$
z^{-1} \rightarrow e^{j \phi} z^{-1}=(\cos \phi+j \sin \phi) z^{-1} .
$$

This transformation rotates the pole-zero plot of a real allpass filter by $\phi$ radians in the z-plane. In practice though, the multipliers which implement $\cos \phi$ and $\sin \phi$ cannot, in general, be quantized such that their squares sum to unity. As such, if the transformation of (3.7) is applied to a minimum multiplier real all-pass filter, the structurally induced lossless property may not be preserved. One useful exception is with $\phi= \pm \pi / 2$, whence (3.7) becomes

$$
z^{-1} \rightarrow \pm j z^{-1} \text {. }
$$

This transformation requires no explicit multipliers, and retains the structurally induced all-pass property. If (3.8) is applied to a first-order real all-pass filter, the resulting com- plex all-pass filter has a pole and zero on the imaginary axis. As shown in Section VI, such structures form useful building blocks in certain multirate filtering systems.

The structures presented here have emphasized the concept of losslessness in the discrete-time domain. We should point out that the connection between discrete-time losslessness (and passivity) and continuous-time lossless (and passive) networks is well established [1]. Thus many of the all-pass filter structures above can be developed as the wave digital counterpart of the appropriate lossless electrical networks. Indeed, a great body of literature has been devoted to this subject; the interested reader is referred to the overview in [19].

\section{Digital Notch Filters}

The first filtering application of the all-pass filter we will investigate is the design of a digital notch filter, which is useful for removing a single-frequency component from a signal, such as an unmodulated carrier in communication systems, or power supply hum from a sampled analog signal, etc. This is easily implemented using the circuit of Fig. 8 , where the transfer function realized is

$$
G(z)=\frac{1}{2}[1+A(z)]
$$

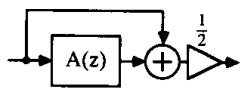

Fig. 8. Implementation of the digital notch filter.

The all-pass function is chosen as second-order, so that the change in phase of $A\left(e^{j \omega}\right)$ as $\omega$ goes from 0 to $\pi$ is $-2 \pi$ radians. As such, from (4.1) it follows that

$$
\begin{aligned}
G\left(e^{j 0}\right) & =G\left(e^{j \pi}\right)=1 \\
G\left(e^{j \omega 0}\right) & =0
\end{aligned}
$$

where $\omega_{0}$ is the angular frequency at which the all-pass filter provides a phase shift of $\pi$ radians. The notch characteristics of (4.2) are structurally induced provided $A(z)$ is realized in a structurally lossless form. Design procedures and a catalogue of minimum-multiplier structures are detailed in [20]. A particularly useful choice for the second-order allpass filter is the lattice filter, shown in minimum multiplier form for convenience in Fig. 9, where

$$
A(z)=\frac{k_{2}+k_{1}\left(1+k_{2}\right) z^{-1}+z^{-2}}{1+k_{1}\left(1+k_{2}\right) z^{-1}+k_{2} z^{-2}} .
$$

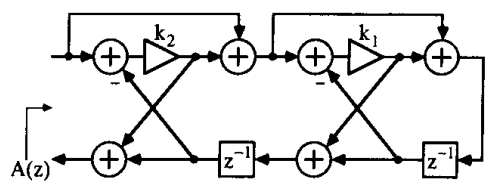

Fig. 9. The second-order all-pass filter $A(z)$ realized using a cascade of single multiplier lattice two-pairs.

One can show that this choice of all-pass filter allows independent tuning of the notch frequency $\omega_{0}$ and the 3-dB attenuation bandwidth $\Omega$ according to

$$
k_{1}=-\cos \omega_{0}
$$




$$
k_{2}=\frac{1-\tan (\Omega / 2)}{1+\tan (\Omega / 2)} .
$$

Note from (4.3) that $k_{2}=r^{2}$, where $r$ is the pole radius. Some frequency response examples are shown in Fig. 10. Fig. 10(a) shows how the 3-dB attenuation bandwidth $\Omega$ can be changed by adjusting only the multiplier $k_{2}$, while Fig. 10(b)

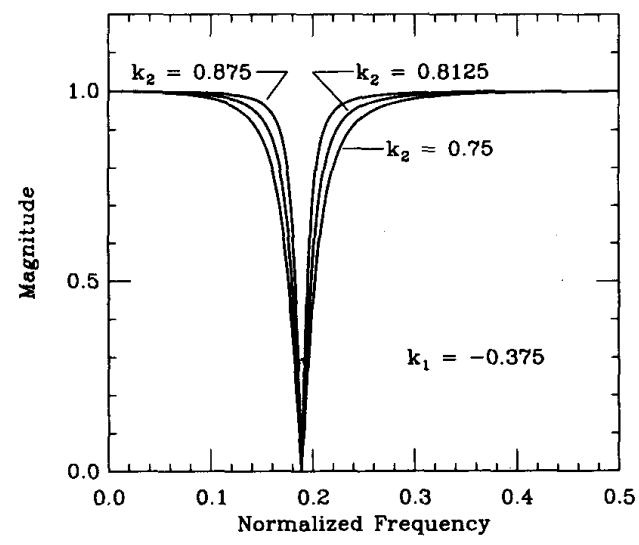

(a)

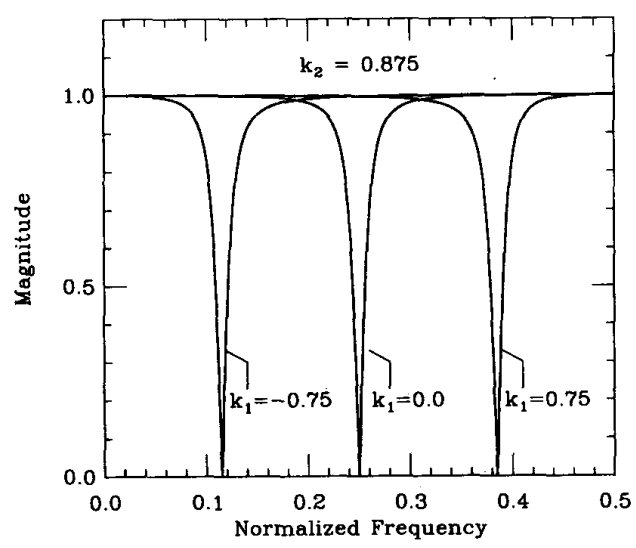

(b)

Fig. 10. The "orthogonal" tuning of the notch filter: (a) illustrates how the rejection bandwidth can be adjusted by varying a single coefficient $k_{2}$, while (b) illustrates how the notch frequency can be tuned by varying only $k_{1}$.

illustrates how the notch frequency $\omega_{0}$ can be changed without affecting the 3-dB attenuation bandwidth by adjusting only $k_{1}$. In view of the "orthogonal" tuning obtained according to (4.4), and the fact that stability is trivial to ensure in a quantized environment, the structure is seen to be quite amenable to adaptive notch filter applications.

An interesting modification to the circuit of Fig. 8 results by replacing the delay variable $z^{-1}$ by $z^{-N}$. The circuit then provides $N$ complex-conjugate zero pairs along the unit circle, located at angles of

$$
\frac{2 \pi n \pm \omega_{0}}{N} \text { radians, } \quad n=0,1, \cdots, N-1,
$$

with $\omega_{0}$ given by (4.4a). Equation (4.4b) holds once $\Omega$ is taken as the total 3-dB attenuation bandwidth; the 3-dB attenuation band about each notch frequency has width $\Omega / N$.
One very useful alignment results with $k_{1}=0$ in (4.4a), which sets $\omega_{0}=\pi / 2$ and places $2 N$ zeros spaced equally along the unit circle. In this case, the frequency $\omega=\pi / 2 N$ and all its odd harmonics are filtered out, which allows the removal of symmetric periodic signals with period $T=4 \mathrm{~N}$ of otherwise arbitrary waveshape.

Greater flexibility in the notch characteristic can be gained by using a first-order complex all-pass filter, with transfer function

$$
A(z)=\mathrm{e}^{j \theta} \frac{\gamma^{*}-z^{-1}}{1-\gamma z^{-1}}
$$

where $\gamma=a+j b$. We begin by writing (4.6) in the form

$$
A(z)=e^{j \theta}\left[G_{1}(z)+j H_{1}(z)\right]
$$

where

$$
\begin{aligned}
& G_{1}(z)=\frac{P(z)}{D(z)}=\frac{a-\left(1+a^{2}-b^{2}\right) z^{-1}+a z^{-2}}{1-2 a z^{-1}+\left(a^{2}+b^{2}\right) z^{-2}} \\
& H_{1}(z)=\frac{Q(z)}{D(z)}=\frac{b-2 a b z^{-1}+b z^{-2}}{1-2 a z^{-1}+\left(a^{2}+b^{2}\right) z^{-2}} .
\end{aligned}
$$

From (4.8) the following observations are noted. First, both $G_{1}(z)$ and $H_{1}(z)$ are second-order real transfer functions with a complex-conjugate pole pair at $z=a \pm j b$, and second, both $P(z)$ and $Q(z)$ are symmetric polynomials, with the zeros of these polynomials determined by the pole locations. Symmetry of $P(z)$ and $Q(z)$ implies the zeros of either polynomial occur as a complex-conjugate pair on the unit circle or as a reciprocal pair on the real axis. Now, by absorbing the factor $\mathrm{e}^{j \theta}$ in (4.7) we obtain

$$
A(z)=G_{2}(z)+j H_{2}(z)
$$

where now

$$
\begin{aligned}
& G_{2}(z)=\frac{\cos \theta P(z)-\sin \theta Q(z)}{D(z)} \\
& H_{2}(z)=\frac{\sin \theta P(z)+\cos \theta Q(z)}{D(z)} .
\end{aligned}
$$

By varying $\theta$, the zeros of $C_{2}(z)$ and $H_{2}(z)$ can be moved along the unit circle (or along the real axis) without affecting the pole locations. As such, low-pass nbtch and high-pass notch characteristics can be realized. In particular, given a pair of poles at $z=a \pm j b$ (which then determines $D(z), P(z)$, and $Q(z))$, the zeros of $G_{2}(z)$ can be placed at $z=e^{ \pm j \omega 0}$ provided that

$$
\frac{P\left(\mathrm{e}^{j \omega 0}\right)}{Q\left(\mathrm{e}^{\mathrm{j \omega 0}}\right)}=\tan \theta .
$$

Since both $P(z)$ and $Q(z)$ are symmetric polynomials, the lefthand side of (4.11) is real for any $\omega_{0}$, which implies a solution for $\theta$ always exists. The filtering function $C_{2}(z)$ is realized by feeding a real input sequénce to the complex all-pass filter $A(z)$ and retaining the real component of the output sequence. The application of such filters in low-sensitivity cascade realizations of elliptic transfer functions can be found in [21].

\section{Doubly COMplementary Filters}

Two filters are said to be complementary if the passbands of one match the stopbands of the other. Complementary filters find applications in various signal processing sys- 
tems where different frequency bands are to be processed separately to measure signal strengths in each band or to achieve, for example, compression or noise reduction. In this section we consider two stable transfer functions which are all-pass complementary

$$
\left|G\left(e^{j \omega}\right)+H\left(e^{j \omega}\right)\right|=1, \quad \text { for all } \omega
$$

as well as power complementary

$$
\left|G\left(e^{j \omega}\right)\right|^{2}+\left|H\left(e^{j \omega}\right)\right|^{2}=1, \quad \text { for all } \omega .
$$

Transfer function pairs which satisfy both complementary properties in (5.1) and (5.2) are termed doubly complementary [22]. We shall see that all-pass filters play a natural role in doubly complementary filters.

First, by combining (5.1) and (5.2) we obtain

$$
\begin{gathered}
\left|G\left(e^{j \omega}\right)+H\left(e^{j \omega}\right)\right|^{2}=\left|G\left(e^{j \omega}\right)\right|^{2}+\left|H\left(e^{j \omega}\right)\right|^{2}=1, \\
\text { for all } \omega .
\end{gathered}
$$

Now, by interpreting $G\left(e^{j \omega}\right)$ and $H\left(e^{j \omega}\right)$ as phasors in the complex plane, application of the law of cosines to the left equality of (5.3) reveals that $G\left(e^{j \omega}\right)$ and $H\left(e^{j \omega}\right)$ must exhibit phase quadrature with respect to each other for all $\omega$. As such, the phasors $\left[G\left(\mathrm{e}^{j \omega}\right)+H\left(\mathrm{e}^{j \omega}\right)\right]$ and $\left[G\left(\mathrm{e}^{j \omega}\right)-H\left(\mathrm{e}^{j \omega}\right)\right]$ must exhibit the same magnitude, namely unity, for all $\omega$. By analytic continuation we thus obtain

$$
\begin{aligned}
& G(z)+H(z)=A_{1}(z) \\
& G(z)-H(z)=A_{2}(z)
\end{aligned}
$$

where $A_{1}(z)$ and $A_{2}(z)$ are stable all-pass functions. Solving for $G(z)$ and $H(z)$ results in

$$
\begin{aligned}
& G(z)=\frac{1}{2}\left[A_{1}(z)+A_{2}(z)\right] \\
& H(z)=\frac{1}{2}\left[A_{1}(z)-A_{2}(z)\right] .
\end{aligned}
$$

A structural implementation of (5.5) appears as Fig. 11, in the form of the sum and difference of two all-pass filters. Referring to Fig. 11, it is seen that, as $G(z)$ is the sum of two all-pass functions, the passband (respectively, stopband) of $G\left(e^{j \omega}\right)$ occurs for frequencies for which the two all-pass functions are in phase (respectively, out of phase) with each other. Since $H(z)$ is the difference of the two all-pass filters, $H\left(\mathrm{e}^{j \omega}\right)$ has a stopband where $G\left(\mathrm{e}^{j \omega}\right)$ has a passband, and vice versa, in accordance with (5.2).

The structure of Fig. 11 was first recognized in digital filter design in terms of wave digital lattice filters [23], whereby bilinearly transformed versions of $G(z)$ and $H(z)$ are interpreted as the reflection and transmission coefficients, respectively, of a real symmetric lossless two-port network with equal resistive terminations. By exploiting certain analogies with classical network theory, Gazsi [24] has developed explicit formulas for the multiplier coefficients of the all-pass filters to implement odd-ordered classical low-pass filter approximations.

The structure of Fig. 11 can also be examined in terms of direct $z$-domain design techniques [10], [22], [25]. For example, Liu and Ansari [10] have shown optimized bandpass filter designs which cannot be derived from classical lowpass filter approximations. We briefly review some design considerations in what follows.

Let $A_{1}(z)$ be an $M_{1}$ th real order all-pass function. Let $A_{2}(z)$ be an $M_{2}$ th real order all-pass function, and in phasor

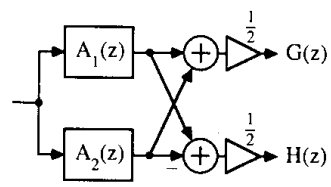

Fig. 11. Implementation of the doubly complementary filter pair as the sum and difference of all-pass functions.

notation let

$$
\begin{aligned}
& A_{1}\left(\mathrm{e}^{j \omega}\right)=\mathrm{e}^{j \phi_{1}(\omega)} \\
& A_{2}\left(\mathrm{e}^{j \omega}\right)=\mathrm{e}^{j \phi_{2}(\omega)}
\end{aligned}
$$

where $\phi_{1}(\omega)$ and $\phi_{2}(\omega)$ are monotone decreasing functions of $\omega$, with $\phi_{1}(0)=\phi_{2}(0)=0, \phi_{1}(\pi)=-M_{1} \pi$, and $\phi_{2}(\pi)=-M_{2} \pi$. Note that this implies for the dc values of the transfer functions $G\left(e^{j 0}\right)=1$ and $H\left(e^{j 0}\right)=0$. Then from (5.5) we obtain

$$
\begin{aligned}
& \left|G\left(e^{j \omega}\right)\right|=\frac{1}{2}\left|e^{j\left[\phi_{1}(\omega)-\phi_{2}(\omega)\right]}+1\right| \\
& \left|H\left(e^{j \omega}\right)\right|=\frac{1}{2}\left|e^{j\left[\phi_{1}(\omega)-\phi_{2}(\omega)\right]}-1\right| .
\end{aligned}
$$

In particular, at $\omega=\pi$

$$
\begin{aligned}
& \left|G\left(e^{j \omega}\right)\right|=\frac{1}{2}\left|e^{j\left[M_{1}-M_{2}\right] \pi}+1\right| \\
& \left|H\left(e^{j \omega}\right)\right|=\frac{1}{2}\left|e^{j\left[M_{1}-M_{2}\right] \pi}-1\right|
\end{aligned}
$$

which shows that a low-pass-high-pass complementary pair requires

$$
M_{1}-M_{2}=2 m \pm 1, \quad m=\text { integer. }
$$

But if $m$ is nonzero, this leads to multiple passbands, and so for a low-pass-high-pass pair

$$
M_{1}-M_{2}= \pm 1 \text {. }
$$

Similarly, for a bandpass-bandstop pair

$$
M_{1}-M_{2}= \pm 2 \text {. }
$$

In general, the condition

$$
M_{1}-M_{2}= \pm L
$$

leads to a total of at least $L+1$ passbands (counting those of $G\left(\mathrm{e}^{j \omega}\right)$ plus those of $H\left(\mathrm{e}^{j \omega}\right)$ ). From (5.5) it is clear that $G(z)$ will have the poles of both $A_{1}(z)$ and $A_{2}(z)$, and thus its order will be $M_{1}+M_{2}$. In view of (5.10) then, it is clear that $M_{1}+$ $M_{2}$ must be an odd integer if a low-pass-high-pass complementary pair is desired.

By lifting the restriction that the all-pass filters be real, even-ordered low-pass-high-pass complementary filters can be realized as well [16]. Thus let $A_{1}(z)$ be a complex allpass function such that complex poles appear without their conjugates, and let $A_{2}(z)$ be obtained from $A_{1}(z)$ by replacing each coefficient with its complex conjugate. Accordingly, we have

$$
A_{2}(\mathrm{z})=A_{1}^{*}\left(z^{*}\right) .
$$

Two real transfer functions $G(z)$ and $H(z)$ can then be defined according to

$$
\begin{aligned}
& G(z)=\frac{1}{2}\left[A(z)+A^{*}\left(z^{*}\right)\right] \\
& j H(z)=\frac{1}{2}\left[A(z)-A^{*}\left(z^{*}\right)\right]
\end{aligned}
$$


where the numbered subscript has been dropped from the complex all-pass function. $A(z)$ contains one pole from each complex conjugate pair of $G(z)$, and so its order is half that of $G(z)$. The terms $G(z)$ and $j H(z)$ in (5.14) above can be interpreted as the conjugate symmetric and conjugate antisymmetric parts, respectively, of the complex function $A(z)$ [5]. As such, only one complex all-pass function need be implemented; the real and imaginary parts of the complex output sequence are identified as the outputs of the filters $G(z)$ and $H(z)$, respectively, (Fig. 12). In effect, when processing a real

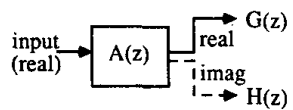

Fig. 12. Separating the real and imaginary outputs of complex all-pass filter to realize an even-ordered complementary fiiter pair.

input sequence, the outputs of the filters $A(z)$ and $A^{*}\left(z^{*}\right)$ are complex conjugates of each other, and thus implementing both complex all-pass filters in (5.14) is redundant. By inverting (5.14) we obtain

$$
\begin{aligned}
& G(z)+j H(z)=A(z) \\
& G(z)-j H(z)=A^{*}\left(z^{*}\right)
\end{aligned}
$$

which shows the complex all-pass complementary property of $G(z)$ and $j H(z)$. With the power complementary relation of (5.2) left intact, $G(z)$ and $j H(z)$ are seen to form a doubly complementary pair.

The realization scheme of Fig. 12 has important practical significance, as one can show that all even order Butterworth, Chebyshev, and elliptic low-pass (and high-pass) transfer functions can be decomposed as per (5.14) [26].

As an illustration, suppose we desire a filter with less that $0.025-\mathrm{dB}$ attenuation for frequencies less than $0.14 \mathrm{rad} / \mathrm{s}$ (normalized frequency), and greater than 45- $\mathrm{dB}$ attenuation for frequencies greater than $0.2 \mathrm{rad} / \mathrm{s}$. It turns out that a sixthorder elliptic transfer function will satisfy these specifications, with the poles of the transfer function located at

$$
z=\left\{\begin{array}{l}
0.468823 \pm j 0.221266 \\
0.475711 \pm j 0.575375 \\
0.501533 \pm j 0.780218
\end{array}\right.
$$

and three zero pairs on the unit circle. Following design procedures in [16], the desired transfer function $G(z)$ can be decomposed into complex all-pass functions as per (5.14), where $A(z)$ is in the form of (2.2) with $M=3$ and

$$
\begin{aligned}
\gamma_{1} & =0.468823+j 0.221266 \\
\gamma_{2} & =0.475711-j 0.575375 \\
\gamma_{3} & =0.501533+j 0.780218 \\
\theta & =0.510542 .
\end{aligned}
$$

A realization of $A(z)$ can be obtained by cascading first order complex all-pass filters as in Fig. 7. A frequency response plot for $G(z)$ appears as Fig. 13. Included on the plot is the frequency response of the transfer function $H(z)$ obtained from the imaginary part of the complex output, which is power complementary to that of $G(z)$, as expected.

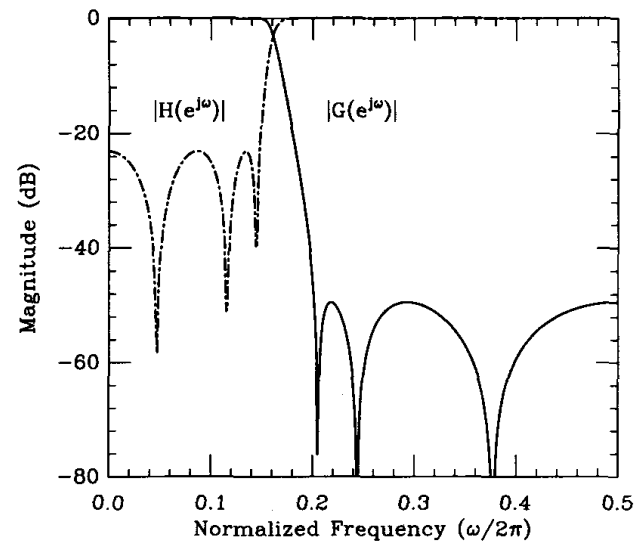

Fig. 13. Frequency responses of the sixth-order complementary filter pair.

\section{Low Sensitivity Properties}

Let us examine the filters of Figs. 11 and 12 in greater detail. With respect to Fig. 11, recall that with $A_{1}(z)$ and $A_{2}(z)$ an $M_{1}$ th- and $M_{2}$ th-order all-pass function, respectively, $G(z)$ has an order $N=M_{1}+M_{2}$. Now, with the all-pass filters realized in a minimum multiplier form, the overall filter requires only $N$ multiplications to compute each output sample, and so is canonic with respect to the number of multiplications. Recall that a direct-form realization would require typically $2 N+1$ multiplications to compute each output sample. But the structure of Fig. 11 in fact makes two complementary transfer functions available, and so represents an optimum in terms of computational efficiency.

The structure of Fig. 12 has similar computational economies. With $G(z)$ an $N$ th-order transfer function, $A(z)$ becomes an N/2th-order complex all-pass function. This allows a filter realization using no more that $N / 2+1 \mathrm{com}-$ plex multiplications to compute each (complex) output sample. If the complex all-pass filter processes a real input sequence, this can be configured as $2 \mathrm{~N}+2$ real multiplications for each complex output sample, but requiring only $N+2$ distinct (real) coefficient values. The number of multiplications is comparable to that of a direct-form realization, and slightly better than that of a cascade realization if the filter order is larger than four. But, as above, a complementary transfer function is made available at no extra cost.

Let us now turn to the sensitivity properties. Referring to (5.5) or (5.14), if the all-pass filters are realized in structurally lossless form, it is clear that $\left|G\left(e^{j \omega}\right)\right|$ can never exceed unity for any value of $\omega$. That is, the magnitude function $\left|C\left(e^{j \omega}\right)\right|$ is structurally bounded above by one. Suppose now that $G(z)$ is designed such that at specific frequencies, to be denoted $\omega_{k}$, the passband amplitude achieves the upper bound of unity, i.e., $\left|G\left(e^{j \omega k}\right)\right|=1$. Regardless of the sign of any multiplier perturbation (due to quantization) the magnitude of the transfer function at $\omega=\omega_{k}$ can only decrease. We can thus apply Orchard's argument [27] at these frequencies (the so-called points of maximum power transfer) to establish the low-passband sensitivity behavior.

Returning to the design example above, Fig. 14(a) shows the passband response obtained for $G(z)$ using an 8-bit binary representation for each multiplier coefficient of the 


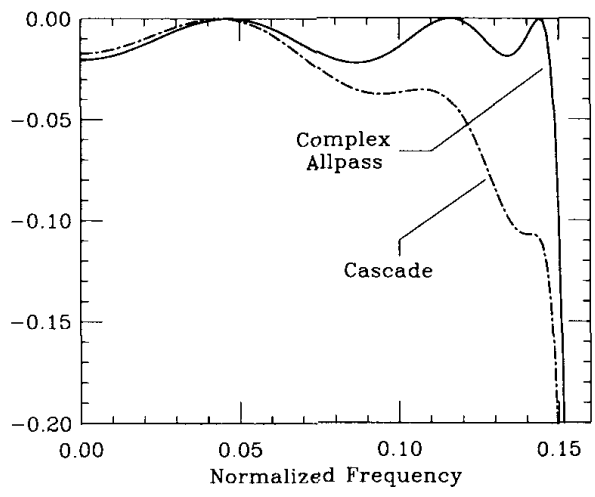

(a)

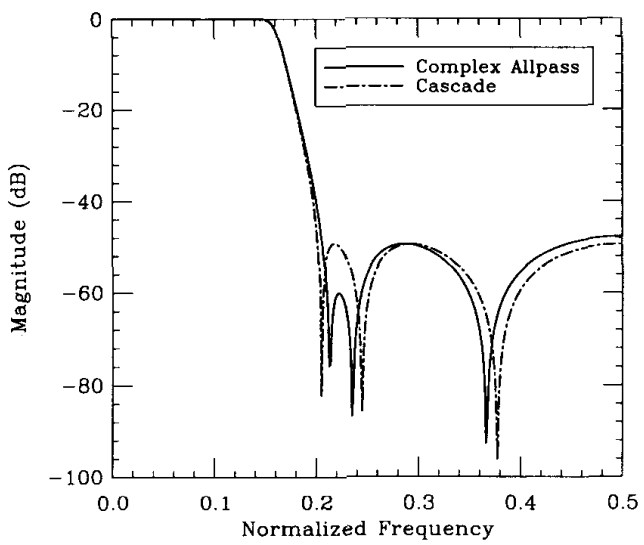

(b)

Fig. 14. Effects of coefficient quantization on $\left|G\left(e^{j \omega}\right)\right|$ using a complex all-pass-based realization and a cascade realization. (a) Passband details and (b) stopband performance.

complex all-pass filter. ${ }^{1}$ Shown for comparison purposes is a conventional cascade realization of $G(z)$ using again 8 bits per multiplier coefficient. The complex all-pass scheme is seen to exhibit much better passband performance. The stopband behavior is superior for the cascade realization though (Fig. 14(b)), as it is known that the cascade structure has very good stopband sensitivity characteristics [28].

\section{Filter Bank Extensions}

Let us rewrite the doubly complementary filter pair of (5.5) as

$$
\left[\begin{array}{l}
G(z) \\
H(z)
\end{array}\right]=\frac{1}{2}\left[\begin{array}{rr}
1 & 1 \\
1 & -1
\end{array}\right]\left[\begin{array}{l}
A_{1}(z) \\
A_{2}(z)
\end{array}\right] .
$$

In matrix form this becomes

$$
\boldsymbol{g}(z)=\frac{1}{2} \boldsymbol{R}_{2} \mathbf{a}(z)
$$

where

$$
\begin{aligned}
& \boldsymbol{g}(z)=[G(z) H(z)]^{T} \\
& \mathbf{a}(z)=\left[A_{1}(z) A_{2}(z)\right]^{T}
\end{aligned}
$$

${ }^{1}$ The quantization is such that the mantissa of a floating-point representation is rounded to the specified number of bits, so that the relative coefficient accuracy does not depend on its magnitude. and $\boldsymbol{R}_{2}$ is two-point DFT matrix. This formulation naturally suggests the following generalization [29]:

$$
g(z)=\frac{1}{N} R_{N} a(z)
$$

where now

$$
\begin{aligned}
& g(z)=\left[\begin{array}{llll}
G_{1}(z) & G_{2}(z) & \cdots & G_{N}(z)
\end{array}\right]^{T} \\
& \mathbf{a}(z)=\left[\begin{array}{llll}
A_{1}(z) & A_{2}(z) & \cdots & A_{N}(z)
\end{array}\right]^{T}
\end{aligned}
$$

and $\boldsymbol{R}_{N}$ is an $\mathrm{N}$-point DFT matrix. The doubly complementary definitions of (5.1) and (5.2) can now be extended to $N$ transfer functions. In particular, the application of Parseval's relation [5] to (5.18) reveals ${ }^{2}$

$$
\begin{aligned}
\sum_{i=1}^{N}\left|G_{i}\left(\mathrm{e}^{j \omega}\right)\right|^{2} & =\tilde{\boldsymbol{g}}\left(\mathrm{e}^{j \omega}\right) \boldsymbol{g}\left(\mathrm{e}^{j \omega}\right)=\frac{1}{N} \tilde{\mathbf{a}}\left(\mathrm{e}^{j \omega}\right) \boldsymbol{a}\left(e^{j \omega}\right) \\
& =\frac{1}{N} \sum_{i=1}^{N}\left|A_{i}\left(e^{j \omega}\right)\right|^{2}=1
\end{aligned}
$$

which shows the power complementary property of the $N$ transfer functions. Also, by exploiting the properties of DFT matrices, it is easily verified that

$$
\sum_{i=1}^{N} G_{i}(z)=A_{1}(z)
$$

which shows the all-pass complementary property. Moreover, the low-passband sensitivity argument above remains intact.

One attractive design strategy is to obtain the all-pass function elements of $a(z)$ from a polyphase decomposition of an Nth-band low-pass filter [30]-[32]. In this case, a(z) takes the form

$$
a(z)=\left[A_{1}\left(z^{N}\right) z^{-1} A_{2}\left(z^{N}\right) z^{-2} A_{3}\left(z^{N}\right) \cdots z^{-N+1} A_{N}\left(z^{N}\right)\right]^{T} .
$$

The transfer functions elements of $\boldsymbol{g}(z)$ in $(5.18 \mathrm{a})$ can be shown to form a uniform filter bank [33] (i.e., the frequency response for each band is a frequency shifted version of that for the adjacent band). Such filter banks are computationally efficient in applications requiring the output signals to be decimated [32]. In addition, computer-aided design procedures for choosing the all-pass functions in (5.21) have been reported [31], [32].

Although in (5.16) we have interpreted $\boldsymbol{R}_{2}$ as a DFT matrix, other unitary matrix families reduce to the same $\boldsymbol{R}_{2}$ in the $2 \times 2$ case [34], [35]. As such, using the $N$-band extension of (5.18), doubly complementary filter banks can be developed for $\boldsymbol{R}_{N}$ chosen as virtually any $N \times N$ unitary matrix.

\section{Multirate Applications}

In the coding and transmission of signals, it is often convenient to split a signal into its various subband components so that perceptual and statistical properties which differ in each frequency band can be exploited separately. This technique is termed subband coding [33], [36]. The basic two-band system is depicted in Fig. 15, where the filters $G(z)$ and $H(z)$ are chosen typically as half-band low-pass and highpass filters, respectively. Since the signal at each analysis filter bank output occupies only half the available digital

${ }^{2}$ The tilde notation indicates the conjugate transpose operation. 


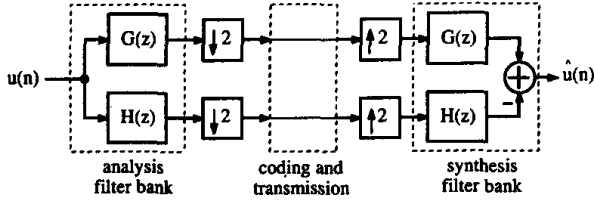

Fig. 15. Basic two-channel maximally decimated subband coding system.

bandwidth, every other sample may be discarded at the output, thereby minimizing the system bit rate. The signals are then coded, transmitted to the receiver, and decoded. In the synthesis filter bank stage, the signals are up-sampled, filtered, and summed into a composite output.

In a practical system, the nonperfect frequency separation of the analysis bank filter pair results in aliasing distortion upon discarding every other sample. This aliasing distortion then propagates through the remainder of the system, and is considered a rather objectionable by-product of the decimation operation. In the absence of coding or transmission errors, straightforward analysis reveals the composite output may be expressed as

$$
\begin{aligned}
\hat{U}(z)= & \frac{1}{2} U(z)\left[G^{2}(z)-H^{2}(z)\right] \\
& +\frac{1}{2} U(-z)[G(z) G(-z)-H(z) H(-z)] .
\end{aligned}
$$

The first term on the right-hand side of (6.1) is a filtered version of the desired signal, while the second term represents the aliasing distortion. Consider now the following choice of filtering functions:

$$
\begin{aligned}
& G(z)=\frac{1}{2}\left[A_{1}\left(z^{2}\right)+z^{-1} A_{2}\left(z^{2}\right)\right] \\
& H(z)=\frac{1}{2}\left[A_{1}\left(z^{2}\right)-z^{-1} A_{2}\left(z^{2}\right)\right]
\end{aligned}
$$

where $A_{1}(\cdot)$ and $A_{2}(\cdot)$ are real all-pass functions. Note that (6.2) implies $H(z)=G(-z)$, so that the frequency responses of the filter pair form a "mirror image" about $\omega=\pi / 2$. This, combined with the phase quadrature condition pointed out in Section V, leads to the name Quadrature Mirror Filters (QMF) [36], [37]. Butterworth and symmetric elliptic halfband filter pairs, for example, can be decomposed as per (6.2), and thus belong to the class of quadrature mirror filters.

This choice of filter pair has the following significance. First, using $H(z)=G(-z)$ it follows that

$$
\begin{aligned}
G(z) & G(-z)-H(z) H(-z) \\
= & G(z) G(-z)-G(-z) G(z)=0 .
\end{aligned}
$$

As such, the aliased signal term in (6.1) vanishes. This result is important because, despite critical subsampling of the analysis filter bank outputs, the synthesis bank output signal is completely free of aliasing effects. Note also that this result makes no assumption as to the quality of frequency selectivity provided by the analysis bank filter pair. ${ }^{3}$

Next, with the aliasing effects removed, the overall system becomes linear and shift-invariant with transfer

${ }^{3}$ In practice though, any reduction in the transmitted bit rate via the coding stage is very dependent on adequate frequency selectivity of the analysis bank filter pair. function

$$
\begin{aligned}
\frac{\hat{U}(z)}{U(z)} & =\frac{1}{2}\left[G^{2}(z)-H^{2}(z)\right] \\
& =\frac{1}{2}[G(z)+H(z)][G(z)-H(z)] \\
& =\frac{1}{2} z^{-1} A_{1}\left(z^{2}\right) A_{2}\left(z^{2}\right) .
\end{aligned}
$$

The last substitution in (6.4) is easily verified using (6.2). The overall transfer function of the multirate system is thus an all-pass function (save for the scale factor error of $\frac{1}{2}$ ), which indicates perfect magnitude reconstruction of the original signal from its critically sampled subbands. Moreover, with the all-pass filters realized in a structurally lossless form, the aliasing cancellation and perfect magnitude reconstruction properties become structurally induced.

The resulting system may be redrawn as Fig. 16(a). Note that the terms $A_{1}\left(z^{2}\right)$ and $A_{2}\left(z^{2}\right)$ are even functions of $z$. As such, multirate identities [33] allow the decimators and interpolators to be passed through the all-pass filters, while replacing $z^{2}$ by $z$ (Fig. 16(b)), which leads finally to the polyphase arrangement of Fig. 16(c). The polyphase structure

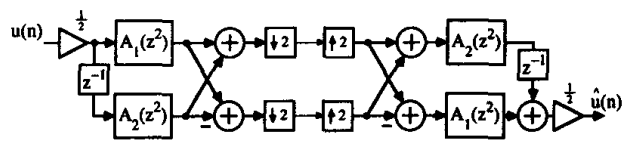

(a)

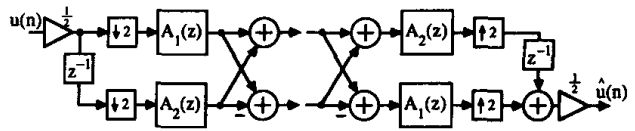

(b)

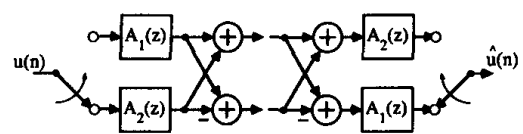

(c)

Fig. 16. (a) Two-band maximally decimated quadraturemirror filter system using all-pass filters. (b) Equivalent system obtained by passing the decimators and interpolators through the all-pass functions. (c) The polyphase implementation.

is attractive because it allows the all-pass filters to operate at the reduced sampling rate. Further design considerations may be found in [38].

Recall from Section $V$ that low-pass-high-pass filter pairs must be of odd order to be realized as per (5.5) (or (6.2)). For the sake of completeness, it is of interest to develop a corresponding result for even ordered low-pass-high-pass pairs. To this end, consider the modified two-channel multirate scheme of Fig. 17 [39], where the analysis filter bank output signals are now subsampled alternately. For this sys-

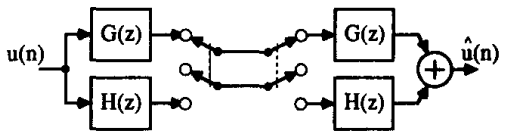

Fig. 17. Modified two-band multirate system using evenordered filters $G(z)$ and $H(z)$ 
tem, the output signal is found as

$$
\begin{aligned}
\hat{U}(z)= & \frac{1}{2} U(z)\left[G^{2}(z)+H^{2}(z)\right] \\
& +\frac{1}{2} U(-z)[G(z) G(-z)-H(z) H(-z)] .
\end{aligned}
$$

In this case, $G(z)$ and $H(z)$ are chosen according to [16]

$$
\begin{aligned}
& G(z)=\frac{1}{2}\left[A(z)+A^{*}\left(z^{*}\right)\right] \\
& H(z)=\frac{1}{2 j}\left[A(z)-A^{*}\left(z^{*}\right)\right]
\end{aligned}
$$

where $A(z)$ is a complex all-pass function which satisfies

$$
A(-z)= \pm j A^{*}\left(z^{*}\right)
$$

The constraint of (6.7) ensures that $H(z)=\mp G(-z)$, so that the aliasing term of (6.5) vanishes. The transfer function of the overall system then becomes

$$
\begin{aligned}
\frac{\hat{U}(z)}{U(z)} & =\frac{1}{2}\left[G^{2}(z)+H^{2}(z)\right] \\
& =\frac{1}{2}[G(z)+j H(z)][G(z)-j H(z)] \\
& =\frac{1}{2} A(z) A^{*}\left(z^{*}\right)
\end{aligned}
$$

which represents a real all-pass function. As above, aliasing distortion is eliminated and the system provides perfect magnitude reconstruction of the critically sampled subbands. A realization scheme incorporating (6.6) is shown as Fig. 18.

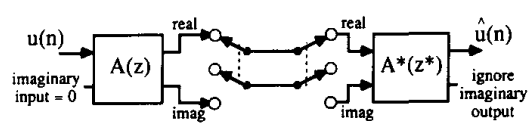

Fig. 18. Implementation of Fig. 17 using complex all-pass filters.

An illustrative example can be developed with the aid of design procedures contained in [16]. In particular, a sixthorder symmetric elliptic transfer function pair is chosen for the analysis filter bank. The transfer function pair $G(\mathrm{z})$ and $H(z)$ are obtained via (6.6) where

$$
\begin{aligned}
A(z)= & e^{j \pi / 4} \frac{z^{-1}+j 0.261935}{1-j 0.261935 z^{-1}} \\
& \cdot \frac{z^{-1}-j 0.674524}{1+j 0.674524 z^{-1}} \frac{z^{-1}+j 0.912402}{1-j 0.912402 z^{-1}} .
\end{aligned}
$$

The frequency response for this symmetric elliptic filter pair is shown as Fig. 19.

Note that the poles of $A(z)$ lie along the imaginary axis, which can be shown to be a necessary consequence of the QMF conditions [16]. Each term in the product of (6.9) can be implemented using a first-order real all-pass function upon applying the frequency transformation $z^{-1} \rightarrow j z^{-1}$ of (3.8) [40]. A complex signal flowgraph of the analysis filter bank appears as Fig. 20. To save on multipliers, the factor $e^{j \pi / 4}=(1+j) / \sqrt{2}$ is implemented in a multiplierless "denormalized" form. The resulting analysis filter bank requires only three multipliers to compute each complex output

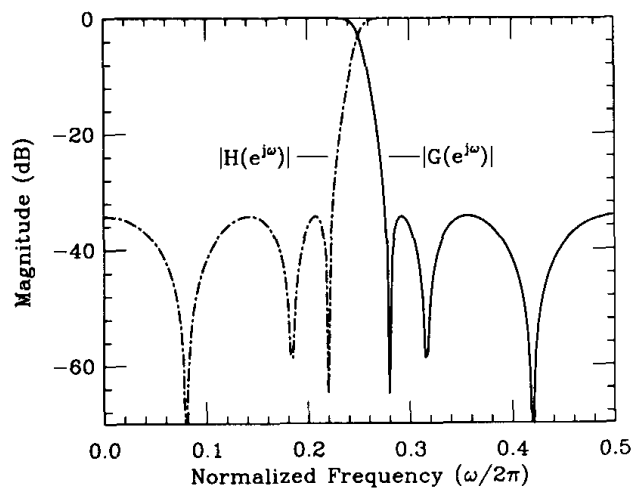

Fig. 19. Frequency responses of a sixth-order symmetric elliptic half-band filter pair.

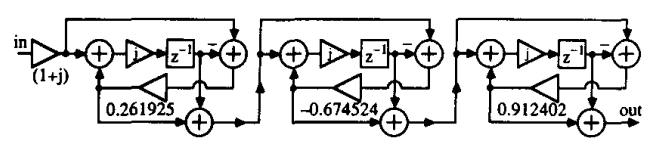

Fig. 20. Implementation of the half-band filter pair as the cascade of three first-order complex all-pass filters. The signals at all nodes are in general complex valued.

sample. Each coefficient is real valued and operates on complex data, and so $G(z)$ and $H(z)$ are simultaneously implemented using only six real multiplications. The corresponding synthesis filter bank can be obtained from the analysis filter bank by replacing $j$ everywhere with $-j$.

\section{Multirate Hilbert Transform Systems}

Suppose now that we have a real half-band filter pair $[G(z)$ $H(z)]$, to which we apply the frequency transformation of (3.8)

$$
\begin{aligned}
& \bar{G}(z)=G(-j z) \\
& \bar{H}(z)=H(-j z) .
\end{aligned}
$$

If $G(z)$ is a half-band low-pass filter with its passband on the "right half" of the unit circle, $\bar{G}(z)$ in $(6.10)$ becomes a complex half-band filter with its passband on the upper half of the unit circle. Such a filter satisfies the frequency-domain constraints of a Hilbert transform system [5]. If $G(z)$ and $H(z)$ form a real half-band filter pair as per (6.2), we obtain for $\bar{G}(z)$ and $\bar{H}(z)$

$$
\begin{aligned}
& \bar{G}(z)=\frac{1}{2}\left[A_{1}\left(-z^{2}\right)+j z^{-1} A_{2}\left(-z^{2}\right)\right] \\
& \bar{H}(z)=\frac{1}{2}\left[A_{1}\left(-z^{2}\right)-j z^{-1} A_{2}\left(-z^{2}\right)\right]
\end{aligned}
$$

where $A_{1}\left(-z^{2}\right)$ and $A_{2}\left(-z^{2}\right)$ remain real all-pass functions. A real-equivalent signal flowgraph for the complex filter $\bar{C}(z)$ appears as Fig. 21. If the input signal is real-valued, Fig. 21 reduces to a pair of all-pass filters which exhibit phase quadrature over the passband region of the complex transfer function $\bar{G}\left(e^{j \omega}\right)$.

Note that the filter pair of (6.11) inherits the property $\bar{H}(z)$ $=\bar{G}(-z)$. Hence, if the filter pair is configured into the multirate system of Fig. 15, aliasing distortion is absent from the output according to (6.3) (upon replacing $G(z)$ and $H(z)$ with their barred versions). The overall transfer function for the 


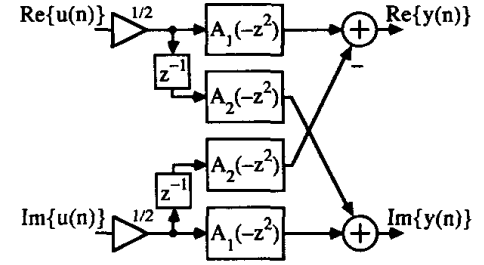

Fig. 21. Implementation of a complex half-band filter using real all-pass filters. If the input signal is real-valued, the system reduces to a parallel all-pass network Hilbert transform system.

system becomes

$$
\begin{aligned}
\frac{\hat{U}(z)}{U(z)} & =\frac{1}{2}\left[\bar{G}^{2}(z)-\bar{H}^{2}(z)\right] \\
& =\frac{1}{2} j z^{-1} A_{1}\left(-z^{2}\right) A_{2}\left(-z^{2}\right)
\end{aligned}
$$

which is an all-pass function as expected.

If the input signal is real, further simplifications are possible, as (6.11) reveals

$$
\bar{H}(\mathrm{z})=\bar{G}^{*}\left(z^{*}\right) \text {. }
$$

As such, the two channels of Fig. 15 process signals which, at each stage, share a complex conjugate relation, with the output formed from the difference of these two channels. This allows one channel of the complex multirate system to be discarded, with the imaginary output from the remaining channel retained as the desired (real) output signal. Using Fig. 21 as the model for $\bar{G}(z)$, the real-equivalent signal flowgraph of Fig. 22(a) is obtained. The analysis bank output signals may be interpreted as the real and imaginary parts of a complex "analytic" sequence with only positive frequency components. A polyphase arrangement is readily obtained as Fig. 22(b). This example thus reaffirms the feasibility of downsampling complex analytic signals [41], [42].

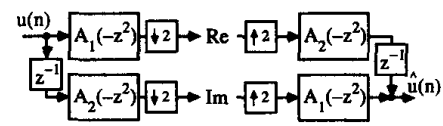

(a)

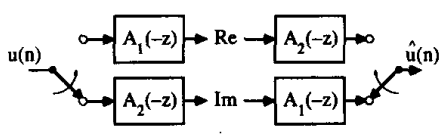

(b)

Fig. 22. (a) Multirate Hilbert transform system offering no aliasing distortion and perfect magnitude reconstruction of the input spectrum. (b) Equivalent polyphase system.

Although we have derived a Hilbert transform system from a real half-band filter pair, it should be pointed out that the all-pass functions in the filter pair of (6.11) can also be obtained using the design formulas in [43]. Several other authors have also discussed design techniques for obtaining Hilbert transform systems using all-pass sections [44][46]. As explained in [42], many of these designs can be identically obtained by applying the transformation $z^{-1} \rightarrow$ $-j z^{-1}$ to known real half-band filter designs. This is an extension of a result by Jackson [47] showing that the approximation problem for Hilbert transformers is closely related to that for half-band low-pass filters. This has been exemplified in (6.10) for half-band filters obtained as the sum and difference of real all-pass functions, although analogous results can be developed for those obtained from complex all-pass functions as per (6.6), as well as half-band designs obtained from approximately linear phase filters (to be introduced in Section VIII). The resulting designs have passbands which are symmetric about $\omega=\pi / 4$, and multirate results in each case can be developed [42]. Finally, we point out that Hilbert transform systems with passbands that are not symmetric about $\omega=\pi / 4$ can be obtained from the above-mentioned designs by using standard frequency transformations [48]. To such systems, however, multirate results are not applicable.

\section{Tunable Filters and Generalized Complementary FILTERS}

Let us return to the doubly complementary filter of Fig. 11 , and consider the filter pair which results from choosing

$$
\begin{aligned}
& A_{1}(z)=1 \\
& A_{2}(z)=\frac{k_{2}+k_{1}\left(1+k_{2}\right) z^{-1}+z^{-2}}{1+k_{1}\left(1+k_{2}\right) z^{-1}+k_{2} z^{-2}} .
\end{aligned}
$$

The transfer function $G(z)$ is recognized as the digital notch filter of Section IV. In view of the power complementary relation of (5.2), $H(z)$ must be a bandpass filter with center frequency $\omega_{0}=\cos ^{-1}\left(-k_{1}\right)$. Consider now the transfer function formed with the following linear combination:

$$
F(z)=G(z)+K H(z) .
$$

A realization is sketched in Fig. 23. Due to the phase quadrature relationship between $G\left(e^{j \omega}\right)$ and $H\left(e^{j \omega}\right)$, it is easily

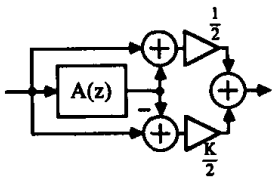

Fig. 23. Tunable frequency response equalization filter.

shown that

$$
\left|F\left(e^{j \omega}\right)\right|^{2}=\left|G\left(e^{j \omega}\right)\right|^{2}+K^{2}\left|H\left(e^{j \omega}\right)\right|^{2} .
$$

In view of the filter type realized for $G(z)$ and $H(z),(7.3)$ shows that the frequency response $F\left(e^{j \omega}\right)$ modifies the gain about the center frequency of $H\left(e^{j \omega}\right)$ while leaving the remainder of the spectrum unaffected. Such a filter is ideally suited as a magnitude equalization filter [49], and finds extensive application in digital audio systems [50]-[52] to compensate for frequency response deficiencies in an acoustic playback environment.

Design equations can be developed as an extension of those presented in (4.4). Thus let $\omega_{0}$ be the frequency about which boost or cut in the magnitude response is desired, and let $\Omega$ be the 3-dB notch bandwidth obtained for $K=0$. The design equations become

$$
k_{1}=-\cos \omega_{0}
$$




$$
\begin{aligned}
k_{2} & =\frac{1-\tan (\Omega / 2)}{1+\tan (\Omega / 2)} \\
K & =F\left(\mathrm{e}^{j \omega 0}\right) .
\end{aligned}
$$

It can also be shown that with $K>0$, the circuit provides minimum phase equalization [49]. Some frequency response examples are shown in Fig. 24, which demon-

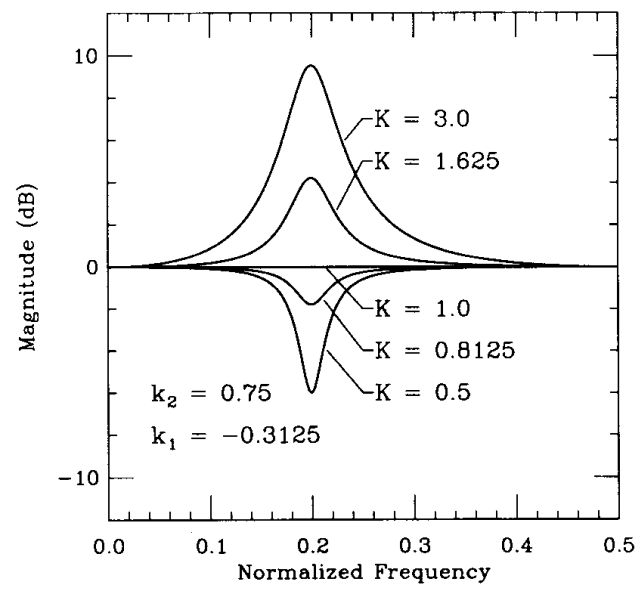

(a)

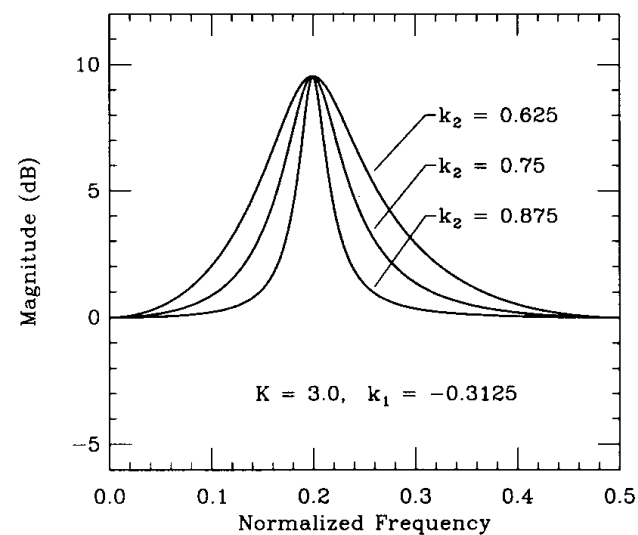

(b)

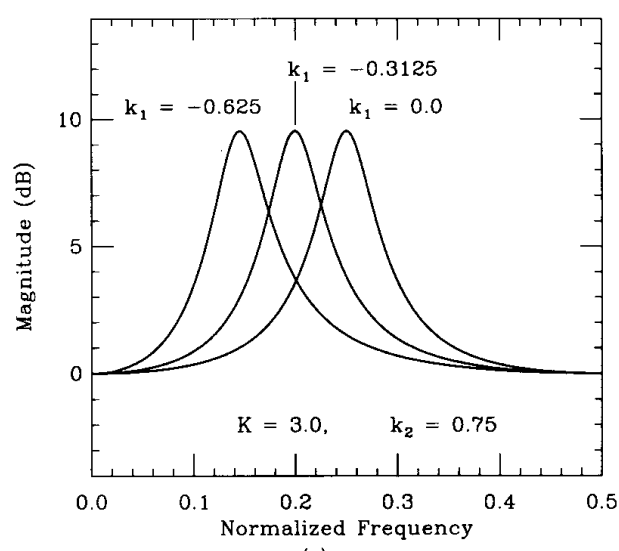

(c)

Fig. 24. Illustrating the parametric adjustment of the frequency response. (a) Variable gain at the center frequency, (b) adjusting the modification bandwidth, and (c) tuning the center frequency. strate the true parametric tuning ability of the circuit. By cascading a few such circuits, a complete parametrically adjustable digital frequency response equalizer may be realized. By comparison, the designs in [50]-[52] require precomputing the multiplier coefficient values for all desired equalizer settings. This does not represent a tunable design, and as such has the drawback of requiring excessive coefficient storage.

More general tunable filters can be realized by using a less trivial choice than (7.1) for the all-pass functions. Such a tunable filter realization is attractive since it allows both the poles and zeros of an Nth-order transfer function $G(z)$ to be tuned by varying only $N$ coefficients in the all-pass filters. If the all-pass filters are realized in lattice form, stability is trivial to ensure simply by constraining the lattice parameters to have magnitudes less than unity. Moreover, the frequency response type (i.e., low-pass, high-pass, bandpass, etc.) is related to the orders of the all-pass filters in view of the discussion surrounding (5.10)-(5.12). If, for example, $A_{1}(z)$ and $A_{2}(z)$ have orders which differ by one, then we are guaranteed in some sense a low-pass characteristic (though not always optimal) for any choice of the all-pass filter parameters, assuming of course that stability is not impaired.

The next problem is to determine the tuning algorithms for the all-pass filter coefficients to achieve the desired tunability of $G(z)$. Suppose that $A_{1}(z)$ and $A_{2}(z)$ in (5.2) are determined so that their sum $G(z)$ is a satisfactory low-pass filter with cutoff frequency $\omega_{1}$. A new low-pass filter with a different cutoff frequency can be obtained from $G(z)$ using the frequency transformations of Constantinides [48]

$$
z^{-1} \rightarrow \beta(z)
$$

where $\beta(z)$ is a stable all-pass function, so that the unit circle in the $z$-plane maps to the unit circle in the $\beta(z)$-plane. Thus by writing $\beta\left(e^{j \omega}\right)=e^{-j \phi(\omega)}$, the transformation of (7.5) may be understood as the frequency mapping

$$
\omega \rightarrow \phi(\omega) \text {. }
$$

Procedures for selecting $\beta(z)$ can be found in many texts on digital filters [5], [28]. The important point for our discussion is that the transformation of (7.5) maps an all-pass function to an all-pass function. Hence if $G(z)$ is the sum of two allpass functions, $G\left(\beta\left(z^{-1}\right)\right)$ is also the sum of two all-pass functions. Thus the problem of tunability is "solved" by implementing the all-pass functions $A_{1}\left(\beta\left(z^{-1}\right)\right)$ and $A_{2}\left(\beta\left(z^{-1}\right)\right)$ in (5.5). In practice though, the direct implementation of (7.5) may lead to delay-free loops if $\beta(z)$ does not contain a pure delay factor. For example, a low-pass-tolow-pass transformation results for [48]

$$
z^{-1} \rightarrow \beta(z)=\frac{z^{-1}-\alpha_{1}}{1-\alpha_{1} z^{-1}}
$$

with

$$
\alpha_{1}=\frac{\sin \left[\left(\omega_{1}-\omega_{2}\right) / 2\right]}{\sin \left[\left(\omega_{1}+\omega_{2}\right) / 2\right]}
$$

where $\omega_{2}$ is the new desired cutoff frequency. Substituting (7.7a) directly for each delay element in $A_{1}(z)$ and $A_{2}(z)$ would introduce delay-free loops. One remedy is to express the coefficients of $A_{1}\left(\beta\left(z^{-1}\right)\right)$ and $A_{2}\left(\beta\left(z^{-1}\right)\right)$ as functions of the variable $\alpha_{1}$. The resulting coefficients can each be expanded 
as a Taylor series in $\alpha_{1}$. If $\alpha_{1}$ is small (corresponding to a small shift in the cutoff frequency), each series expansion may be truncated after the linear term to obtain a simplified expression for the coefficients [53]. For example, with

$$
A_{1}(z)=\frac{z^{-1}-a_{0}}{1-a_{0} z^{-1}}
$$

we find

$$
A_{1}\left(\beta\left(z^{-1}\right)\right)=\frac{z^{-1}-\left(a_{0}+\alpha_{1}\right) /\left(1+\alpha_{1} a_{0}\right)}{1-\left(a_{0}+\alpha_{1}\right) /\left(1+\alpha_{1} a_{0}\right) z^{-1}} .
$$

By expanding the coefficient $\left(a_{0}+\alpha_{1}\right) /\left(1+\alpha_{1} a_{0}\right)$ in a Taylor series in $\alpha_{1}$ and truncating after the linear term, we obtain the approximation

$$
A_{1}\left(\beta\left(z^{-1}\right)\right) \approx \frac{z^{-1}-\left[a_{0}+\left(1-a_{0}^{2}\right) \alpha_{1}\right]}{1-\left[a_{0}+\left(1-a_{0}^{2}\right) \alpha_{1}\right] z^{-1}}
$$

which can be realized using any all-pass implementation scheme by adding to the multiplier branch of value $a_{0}$ the parallel tuning branch of value $b \alpha_{1}$, where $b=1-a_{0}^{2}$. Likewise, if $A_{2}(z)$ is a second-order all-pass function

$$
A_{2}(z)=\frac{a_{2}-a_{1} z^{-1}+z^{-2}}{1-a_{1} z^{-1}+a_{2} z^{-2}}
$$

a similar procedure results in

$A_{2}\left(\beta\left(z^{-1}\right)\right)$

$\approx \frac{\left[a_{2}+\alpha_{1} a_{1}\left(1-a_{2}\right)\right]-\left[a_{1}+\alpha_{1}\left(2+2 a_{2}-a_{1}^{2}\right)\right] z^{-1}+z^{-2}}{1-\left[a_{1}+\alpha_{1}\left(2+2 a_{2}-a_{1}^{2}\right)\right] z^{-1}+\left[a_{2}+\alpha_{1} a_{1}\left(1-a_{2}\right)\right] z^{-2}}$

Note again that the transfer function coefficients have a tuning branch of the type $b_{i} \alpha_{1}, i=1,2$, in parallel with the nominal multiplier values $a_{1}$ and $a_{2}$. Higher order all-pass functions can be factored into first-order and second-order factors, with the results of (7.10) and (7.12) immediately applicable. Although the derivation of (7.10) and (7.12) assumes a small tuning variation for $\alpha_{1}$, tuning ranges of several octaves have been reported for narrow-band lowpass filters [53].

Another useful choice for $\beta(z)$ is the following low-passto-band-pass transformation:

$$
z^{-1} \rightarrow \beta(z)=-z^{-1} \frac{z^{-1}-\alpha_{2}}{1-\alpha_{2} z^{-1}}
$$

with $\alpha_{2}=-\cos \left(\omega_{3}\right)$, where $\omega_{3}$ is the desired center frequency of the band-pass filter. Note that (7.13) can be directly substituted for each delay element in $A_{1}(z)$ and $A_{2}(z)$ without introducing delay-free loops. This results in a bandpass filter $G\left(\beta\left(z^{-1}\right)\right)$ whose center frequency may be tuned by adjusting a single parameter $\alpha_{2}$.

\section{Generalized Complementary Filters}

Let us remove any constraints from the all-pass functions, and consider now the transfer function pair which results from

$$
\begin{aligned}
& F_{1}(z)=K_{1} G(z)+K_{2} H(z) \\
& F_{2}(z)=K_{2} G(z)-K_{1} H(z) .
\end{aligned}
$$

By exploiting the phase quadrature relationship between $G\left(e^{j \omega}\right)$ and $H\left(e^{j \omega}\right)$ we obtain

$$
\begin{aligned}
& \left|F_{1}\left(e^{j \omega}\right)\right|^{2}=K_{1}^{2}\left|G\left(e^{j \omega}\right)\right|^{2}+K_{2}^{2}\left|H\left(e^{j \omega}\right)\right|^{2} \\
& \left|F_{2}\left(e^{j \omega}\right)\right|^{2}=K_{2}^{2}\left|G\left(e^{j \omega}\right)\right|^{2}+K_{1}^{2}\left|H\left(e^{j \omega}\right)\right|^{2} .
\end{aligned}
$$

Using the fact that $G\left(e^{j \omega}\right)$ and $H\left(e^{j \omega}\right)$ are power complementary, we find

$$
\left|F_{1}\left(e^{j \omega}\right)\right|^{2}+\left|F_{2}\left(\mathrm{e}^{j \omega}\right)\right|^{2}=K_{1}^{2}+K_{2}^{2} .
$$

Such filter pairs may be termed generalized complementary filters. Now, substituting (5.5) into (7.14) results in

$$
\begin{aligned}
{\left[\begin{array}{l}
F_{1}(z) \\
F_{2}(z)
\end{array}\right] } & =\frac{1}{2}\left[\begin{array}{ll}
K_{1}+K_{2} & K_{1}-K_{2} \\
K_{2}-K_{1} & K_{1}+K_{2}
\end{array}\right]\left[\begin{array}{l}
A_{1}(z) \\
A_{2}(z)
\end{array}\right] \\
& =r\left[\begin{array}{cc}
\cos \theta & -\sin \theta \\
\sin \theta & \cos \theta
\end{array}\right]\left[\begin{array}{l}
A_{1}(z) \\
A_{2}(z)
\end{array}\right]
\end{aligned}
$$

where

$$
\begin{aligned}
r^{2} & =\frac{2}{K_{1}^{2}+K_{2}^{2}} \\
\theta & =\tan ^{-1}\left(\frac{K_{2}-K_{1}}{K_{2}+K_{1}}\right) .
\end{aligned}
$$

Note that with $\theta=\pi / 4$ and $r=1 / \sqrt{2}$, the doubly complementary filters of Section $V$ result, save for a minus sign in the second transfer function. Using (7.15) the following is easily inferred:

$$
\max _{\omega}\left|F_{1}\left(\mathrm{e}^{j \omega}\right)\right|=\max _{\omega}\left|F_{2}\left(\mathrm{e}^{j \omega}\right)\right|=\max \left(\left|K_{1}\right|,\left|K_{2}\right|\right) .
$$

Equation (7.18) holds true upon replacing "max" everywhere with " $\min$." As such, if neither $K_{1}$ nor $K_{2}$ is zero, then the transfer function pair provides no stopband per se. However, such filters find application in multi-level passband filters [54], [55], and generalized magnitude equalization [52].

\section{Group-Delay EQualization}

Thus far we have overlooked the obvious implication of (2.1): An all-pass filter can be cascaded with another filter to alter the phase response while leaving the magnitude response unaffected. In this section, we provide an overview of applications to group delay equalization, and call attention to a class of IIR filters which has intrinsically good phase response properties.

Recall from (2.13) that the group-delay response $\tau_{F}(\omega)$ of a given filter $F(z)$ is defined as the negative of the derivative of its phase response

$$
\tau_{F}(\omega)=-\frac{d}{d \omega}\left[\arg F\left(e^{j \omega}\right)\right]
$$

With the transfer function $F(z)$ expressed in rational form

$$
F(z)=\frac{P(z)}{Q(z)}
$$

one can show [56] that the group-delay response becomes

$$
\tau_{F}(\omega)=\operatorname{Re}\left[\frac{z Q^{\prime}(z)}{Q(z)}-\frac{z P^{\prime}(z)}{P(z)}\right]_{z=e^{j \omega}}
$$

where the prime denotes differentiation with respect to $z$. 
Applying (8.3) to an all-pass function

$$
A(z)=\frac{z^{-M} D\left(z^{-1}\right)}{D(z)}
$$

we obtain the result

$$
\tau_{A}(\omega)=M+2 \operatorname{Re}\left[\frac{z D^{\prime}(z)}{D(z)}\right]_{z=e^{j \omega}} .
$$

Our goal then is to choose $A(z)$ such that, when cascaded with $F(z)$, the resulting group-delay function approximates some desired response $E(\omega)$ over the passband interval(s) of $F\left(\mathrm{e}^{j \omega}\right)$. Recalling that the group-delay response is additive in a cascade connection, our problem is equivalent to minimizing the magnitude of the error function

$$
\epsilon(\omega)=E(\omega)-\left[\tau_{F}(\omega)+\tau_{A}(\omega)\right]
$$

over the passband interval(s) of $F\left(e^{j \omega}\right)$ with proper choice of $\tau_{A}(\omega)$. Typically, $E(\omega)$ is chosen as a constant over the passband interval(s) of $F\left(\mathrm{e}^{j \omega}\right)$.

If a minimax approximation to a constant group delay is desired, then one is tempted to appeal to the powerful alternation theorem for rational functions [57]. Roughly stated, this theorem asserts that the unique best optimum $\tau_{A}(\omega)$ approximating $E(\omega)-\tau_{f}(\omega)$ in a Chebyshev (or minimax) sense is found when the error function $\epsilon(\omega)$ in (8.6) alternates in sign from extremum to extremum with equal magnitude. However, as pointed out by Deczky [58], group-delay functions do not satisfy the conditions of the alternation theorem, and as such an equiripple error function does not ensure a minimax solution.

Nonetheless, an equiripple approximation to a constant group delay is attractive in applications where waveform distortion is to be avoided. Unfortunately, the optimization of $\tau_{A}(\omega)$ imposes nonlinear constraints on the parameters of the all-pass function $A(z)$, and closed-form solutions for the all-pass filter parameters are not generally available. As such, one must resort to iterative computer approximation methods. An early report of all-pass filter design for groupdelay equalization using the Fletcher-Powell algorithm was given by Deczky [59]. An improvement in speed using a modified Remez-type exchange algorithm was subsequently reported in [58].

Design procedures for all-pole filters offering an equiripple group-delay response have also been reported in the literature [60]-[62]. These methods can be used for groupdelay equalization following a minor reformulation of the problem. Consider the all-pole counterpart to the all-pass function of (8.4)

$$
B(z)=\frac{1}{D(z)}
$$

The group-delay response $\tau_{B}(\omega)$ corresponding to $B\left(e^{j \omega}\right)$ can be found with the aid of (8.3)

$$
\tau_{B}(\omega)=\operatorname{Re}\left[\frac{z D^{\prime}(z)}{D(z)}\right]_{z=\mathrm{e}^{\prime \omega}}=\frac{1}{2}\left[\tau_{A}(\omega)-M\right] .
$$

Thus let the error function of (8.6) be rewritten as

$$
\frac{1}{2} \epsilon(\omega)=\frac{1}{2}\left[E(\omega)-\tau_{F}(\omega)-M\right]-\tau_{B}(\omega)
$$

We can thus take $\frac{1}{2}\left[E(\omega)-\tau_{F}(\omega)-M\right]$ as the "ideal" groupdelay response, and search for the all-pole filter $B(z)=$
$1 / D(z)$ with the best group-delay approximation, using the methods in [60]-[62]. The all-pass function $A(z)$ obtained from (8.4) then achieves the desired group-delay equalization of $F(z)$.

Consider now one filter from the doubly complementary pair of (5.5)

$$
G(z)=\frac{1}{2}\left[A_{1}(z)+A_{2}(z)\right]
$$

If we let $\tau_{1}(\omega)$ and $\tau_{2}(\omega)$ denote, respectively, the group-delay responses of $A_{1}\left(e^{j \omega}\right)$ and $A_{2}\left(e^{j \omega}\right)$, then using (8.3) reveals, following some algebra, that the group-delay response $\tau_{G}(\omega)$ for $G\left(e^{j \omega}\right)$ becomes

$$
\tau_{C}(\omega)=\frac{\tau_{1}(\omega)+\tau_{2}(\omega)}{2} .
$$

Recall that the passband(s) for $G\left(e^{j \omega}\right)$ occur where $A_{1}\left(e^{j \omega}\right)$ and $A_{2}\left(e^{i \omega}\right)$ are in-phase. Hence, by equalizing the passband group delay of $G\left(e^{j \omega}\right)$, we approximately equalize the groupdelay functions $\tau_{1}(\omega)$ and $\tau_{2}(\omega)$ over this same frequency range. This suggests that good magnitude and group-delay responses can be simultaneously achieved by choosing $A_{1}(z)$ such that its group-delay response is favorable over the desired passband region, and then choosing $A_{2}(z)$ such that it phase response is in-phase and out-of-phase with respect to $A_{1}(z)$ over the passband and stopband regions, respectively.

A slight variation to this strategy results if we consider the phase response, rather than the group-delay response, to be important. Thus let us choose one all-pass function as a pure delay

$$
A_{\uparrow}(z)=z^{-M}
$$

and choose $A_{2}(z)$ to be in-phase and out-of-phase with respect to the delay over the passband and stopband regions, respectively. The passband magnitude and phase characteristics of $G\left(e^{j \omega}\right)$ are simultaneously optimized by forcing $A_{2}\left(\mathrm{e}^{j \omega}\right)$ to approximate a linear phase characteristic over the passband region of $G\left(e^{j \omega}\right)$. Such filters are termed approximately linear phase [63], [64]. Although the order of these filters is typically higher than that of an elliptic filter (with nonlinear phase characteristics) meeting the same frequency-selective specifications [64], the signal delay is typically less than that obtained using an FIR filter. These filters are thus attractive in applications requiring low waveform distortion with small delay.

\section{iX. State-Space Manifestations of the All-Pass Property}

The state-space description provides a powerful framework whereby many concepts of system theory can be addressed in a unified manner. The losslessness property satisifed by all-pass functions induces some elegant properties on a state-space description, which we summarize below in the discrete-time lossless bounded real lemma [65]. Some applications of this lemma to finite word-length effects in digital filters are outlined.

We consider a single-input/single-output (SISO) statespace description

$$
\begin{aligned}
\boldsymbol{x}(n+1) & =\boldsymbol{A x}(n)+\boldsymbol{b} u(n) \\
y(n) & =\boldsymbol{c}^{t} \boldsymbol{x}(n)+d u(n)
\end{aligned}
$$

where $\boldsymbol{A}$ is $N \times N, \boldsymbol{b}$ and $\boldsymbol{c}$ are $N \times 1, d$ is $1 \times 1$, and $\boldsymbol{x}(n)$ $=\left[x_{1}(n) \cdots x_{N}(n)\right]^{t}$ is the state vector. The transfer function 
$Y(z) / U(z)$ is given by

$$
\frac{Y(z)}{U(z)}=d+c^{t}(z I-A)^{-1} b .
$$

We assume also that the realization is minimal in the number of states, so that the order of $Y(z) / U(z)$ is $N$ (i.e., there are no pole-zero cancellations). Now, given any nonsingular $N \times N$ matrix $T$, we can invoke a similarity transformation by replacing the set $\{A, b, c\}$ with

$$
A_{1}=T^{-1} A T \quad b_{1}=T^{-1} b \quad c_{1}^{t}=c^{t} T .
$$

This transformation has no effect on the external transfer function $Y(z) / U(z)$, and with various choices of $T$, we can derive an unlimited number of structures to implement the given transfer function. (In fact, all minimal realizations of a given transfer function are related through a similarity transformation.) The internal properties of the system though, such as scaling at internal nodes, overflow characteristics, roundoff noise, and coefficient sensitivities, can vary markedly among different representations.

If $Y(z) / U(z)$ is an all-pass function, the state-space parameters $\{A, b, c, d\}$ in (9.1) satisfy some very elegant properties which have implications in roundoff noise gain, scaling properties, and limit cycle behavior. We begin by quoting a scalar (SISO) version of the discrete-time lossless bounded real (LBR) lemma:

Lemma [65]: Given the state-space system of (9.1), the transfer function $Y(z) / U(z)$ in (9.2) is a stable all-pass function if and only if there exists an $N \times N$ symmetric positivedefinite matrix $\mathbf{P}$ such that

$$
\begin{aligned}
& A^{t} P A+c c^{t}=P \\
& b^{t} P b+d^{t} d=1 \\
& A^{t} P b+c d=0 .
\end{aligned}
$$

To better understand this lemma, we can derive from (9.4) an important "energy balance" result. Since $\boldsymbol{P}$ is symmetric and positive-definite, there exists a nonsingular matrix $\boldsymbol{I}$ such that $\boldsymbol{P}=\boldsymbol{T}^{t} \boldsymbol{T}$. Consider the new set of state-space parameters which result from the (inverse) similarity transformation

$$
A_{2}=T A T^{-1} \quad b_{2}=T b \quad c_{2}^{t}=c^{t} T^{-1} .
$$

Writing (9.4) in terms of the parameters $\left\{A_{2}, b_{2}, c_{2}, d\right\}$ results in

$$
\begin{aligned}
& A_{2}^{t} A_{2}+c_{2} c_{2}^{t}=I \\
& b_{2}^{t} b_{2}+d^{t} d=1 \\
& A_{2}^{t} b_{2}+c_{2} d=0 .
\end{aligned}
$$

By considering the $(N+1) \times(N+1)$ matrix $R$ formed as

$$
\boldsymbol{R}=\left[\begin{array}{ll}
A_{2} & b_{2} \\
c_{2}^{t} & d
\end{array}\right]
$$

the constraints of (9.6) become equivalent to orthogonality of $\boldsymbol{R}$

$$
R^{t} R=I .
$$

Now, upon recognizing that (9.1) may be written as

$$
\left[\begin{array}{c}
x(n+1) \\
y(n)
\end{array}\right]=R\left[\begin{array}{c}
x(n) \\
u(n)
\end{array}\right]
$$

the orthogonality of $R$ is in turn equivalent to

$$
\begin{gathered}
y^{t}(n) y(n)+x^{t}(n+1) x(n+1) \\
-x^{t}(n) x(n)=u^{t}(n) u(n) .
\end{gathered}
$$

Equation (9.10) states that, at time $n$, the instantaneous output energy $y^{t}(n) y(n)$ plus the instantaneous increase in state energy $\left[x^{t}(n+1) x(n+1)-x(n)^{t} x(n)\right]$ is precisely equal to the instantaneous input energy $u^{t}(n) u(n)$. Hence (9.10) is an energy balance relation. Note that this statement is stronger than that of (2.8); any structure satisfying the energy balance relation certainly satisfies the (external) losslessness condition of (2.8), but the converse is not necessarily true. However, given any lossless structure satisfying (2.8), there exists a similarity transformation which renders the structure in an energy balanced form. (Such a transformation can, in fact, be constructed from the observability grammian of the system [66].) From this observation, we may restate the scalar discrete-time LBR lemma as follows:

$Y(z) / U(z)$ is an all-pass function if and only if it admits a realization whose state-space description satisfies the energy balance relation of (9.10).

Consider, for example, cascaded lattice structure of Fig. 3. By choosing the outputs of the delay variables as the states $x_{k}(n)$, and implementing each two-pair in normalized form (cf. Fig. 4(b)), the structure is known [14], [65] to satisfy the energy balance realization of (9.10). Since an arbitrary stable all-pass function can be realized using the normalized cascaded lattice, we have identified an energy balanced structure. In fact, many more such structures can be identified as, e.g., orthogonal digital filters [67], [68], properly scaled wave digital filters [19], and LBR digital filters [2].

\section{Applications to Roundoff Noise Gain}

The state-space description of (9.1) is depicted in Fig. 25(a). In a practical implementation, quantizers must be introduced into the feedback loop to prevent an unlimited accumulation of the number of bits required to represent the signals. The quantization error is typically modeled by introducing an error vector $\mathbf{e}(n)$ in the feedback loop, as shown in Fig. 25(b). Consider again the cascaded lattice structure of Fig. 3. If one quantizer is inserted after each lattice section just prior to each delay, the model of Fig. 25(b) holds. Since the model does not permit any quantizers inside the lattice sections, a bit accumulation occurs pro-

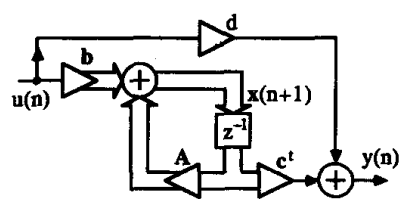

(a)

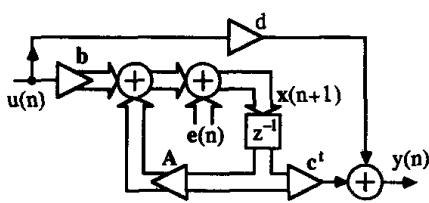

(b)

Fig. 25. (a) State-space filter description. (b) Error vector model for quantization noise. 
gressing from left to right along the upper portion of the lattice, although this accumulation is finite (proportional to $N)$. Such accumulation can be avoided by inserting two quantizers (rather than one) between successive lattice sections. We shall proceed with the model of Fig. 25(b), though, to keep the discussion manageable.

Our goal is to minimize the error component in the output sequence introduced by the quantization error vector $\mathbf{e}(n)$, subject to the constraint that the transfer function to each state variable be properly scaled to minimize the probability of overflow. Let us introduce two vectors

$$
f(n)=\left[f_{1}(n) \cdots f_{N}(n)\right]^{t}
$$

and

$$
g(n)=\left[g_{1}(n) \cdots g_{N}(n)\right]^{t}
$$

such that $f_{k}(n)$ is the response at the $k$ th state variable to a unit pulse input, and $g_{k}(n)$ is the response at the output to a unit pulse at the $k$ th state variable. These vectors are easily found as

$$
f(n)=A^{n} b \quad g^{t}(n)=c^{t} A^{n} .
$$

It is convenient to introduce the controllability and observability Grammian matrices $\boldsymbol{K}$ and $\boldsymbol{W}$ associated with these two vectors:

$$
\begin{aligned}
& \boldsymbol{K}=\sum_{n=0}^{\infty} \boldsymbol{f}(n) \boldsymbol{f}^{t}(n)=\sum_{n=0}^{\infty} A^{n} b\left(A^{n} b\right)^{t} \\
& W=\sum_{n=0}^{\infty} g(n) g^{t}(n)=\sum_{n=0}^{\infty}\left(c^{t} A^{n}\right)^{t}\left(c^{t} A^{n}\right) .
\end{aligned}
$$

These matrices are positive-definite provided there are no pole-zero cancellations in the filter [69], and can be identified as solutions to the equations

$$
\boldsymbol{K}=\boldsymbol{A} \boldsymbol{K} A^{t}+\boldsymbol{b} \boldsymbol{b}^{t} \quad W=A^{t} \boldsymbol{W} A+c c^{t} .
$$

Moreover, if $\boldsymbol{A}$ has all its eigenvalues inside the unit circle, the solutions $K$ and $\boldsymbol{W}$ in (9.13) are unique [70]. In particular, the diagonal elements of either matrix are the squares of the $\ell_{2}$ norms of the elements of $\boldsymbol{f}$ and $g$

$$
\begin{gathered}
K_{k k}=\left\|f_{k}\right\|^{2}=\sum_{n=0}^{\infty}\left|f_{k}(n)\right|^{2} \\
W_{k k}=\left\|g_{k}\right\|^{2}=\sum_{n=0}^{\infty}\left|g_{k}(n)\right|^{2} .
\end{gathered}
$$

Assume now that the components of the noise vector $(n)$ are uncorrelated and that each is white with variance $\sigma_{e}^{2}$, i.e., the covariance matrix of $\mathrm{e}(n)$ is $\sigma_{\mathrm{e}}^{2} I$. Under these assumptions, the variance of the error at the filter output caused by the quantizers is equal to

$$
\sigma_{0}^{2}=\sigma_{e}^{2} \sum_{k=1}^{N} W_{k k}
$$

Now, the diagonal elements $K_{k k}$ of the matrix $K$ represent the square of the $\ell_{2}$ norms of the impulse reponse sequences $f_{k}(n)$ to the state variable nodes. For a structure scaled in an $\ell_{2}$ sense, these quantities should equal unity. This is easily accomplished by using a diagonal similarity transformation matrix $T$, such that

$$
T=\operatorname{diag}\left\{1 /\left\|f_{k}\right\|\right\}
$$

In effect, each term $f_{k}(n)$ is replaced by $f_{k}(n) /\left\|f_{k}\right\|$, and each term $g_{k}(n)$ is replaced by $\left\|f_{k}\right\| g_{k}(n)$ to compensate. Hence, the roundoff noise variance for a scaled realization in terms of the unscaled parameters is

$$
\sigma_{0}^{2}=\sigma_{e}^{2} \sum_{k=1}^{N}\left\|f_{k}\right\|^{2}\left\|g_{k}\right\|^{2}=\sigma_{e}^{2} \sum_{k=1}^{N} K_{k k} W_{k k} .
$$

For a given transfer function, the term

$$
\sum_{k=1}^{N} K_{k k} W_{k k}
$$

depends on the state-space description, and can be considered a "noise gain" for the realization in question. It has been shown [69] that this noise gain is minimized when $\{A, b, c\}$ are such that $K$ and $W$ satisfy the following two properties:

1) $\boldsymbol{K}=\boldsymbol{\Lambda} \boldsymbol{W} \boldsymbol{\Lambda}$ for some diagonal matrix $\boldsymbol{\Lambda}$ of positive elements.

2) $K_{k k} W_{k k}=$ constant independent of $k$.

In fact, a minimum-noise description satisfying these properties always exists [69].

Assume now that $Y(z) / U(z)$ is an all-pass function realized in an energy balanced structure, so that the matrix $R$ in (9.8) is orthogonal. The orthogonality of $\boldsymbol{R}$ also implies orthogonality of $\boldsymbol{R}^{t}$, so that $\boldsymbol{R} \boldsymbol{R}^{t}=I$ yields three equations analogous to (9.6). The first of these is

$$
A A^{t}+b b^{t}=I .
$$

The comparison of (9.19) with (9.13) reveals $K=I$, which indicates an energy balanced filter is inherently scaled in an $\ell_{2}$ sense. Likewise, comparing (9.6a) with (9.13) reveals $W$ $=I$, so that the roundoff noise is minimized according to the conditions (9.18) above. In other words, for any energybalanced structure, the constraints of internal scaling and minimum roundoff noise are automatically satisfied. For such structures, the output noise variance is found from (9.17) as

$$
\sigma_{0}^{2}=\sigma_{\mathrm{e}}^{2} \sum_{k=1}^{N} K_{k k} W_{k k}=N \sigma_{\mathrm{e}}^{2}
$$

so that the noise gain is found knowing only the filter order. We point out that this result holds independent of the pole locations of the filter.

Referring again to the normalized lattice structure, in practice it may be convenient to introduce quantizers into both upper and lower branches connecting adjacent sections to prevent word length from accumulating in the successive lattice stage computations. Upon effecting this modification, the roundoff noise variance appearing at the output can be found as

$$
\sigma_{0}^{2}=2 N \sigma_{\mathrm{e}}^{2} .
$$

The state-space descriptions corresponding to the other Gray-Markel lattice structures can be obtained from that of the normalized form via a diagonal similarity transformation matrix $T$. Accordingly, the matrices $K_{1}$ and $W_{1}$ for such structures are given by

$$
\begin{aligned}
& \boldsymbol{K}_{1}=\boldsymbol{T}_{1}^{-1} \boldsymbol{K} \boldsymbol{T}_{1}^{-t}=\boldsymbol{T}_{1}^{-2} \\
& \boldsymbol{W}_{1}=\boldsymbol{T}_{1}^{t} \boldsymbol{W} \boldsymbol{T}_{1}=\boldsymbol{T}_{1}^{2} .
\end{aligned}
$$


These matrices still satisfy conditions (9.18), which indicates that scaled versions of these structures are minimum noise structures. Of course, upon scaling these structures in an $\ell_{2}$ sense, the resulting state-space description coincides with that of the normalized form.

\section{Limit Cycle Behavior}

The quantizers in the feedback loop of Fig. 25(b) are nonlinear elements, and can cause nonlinear oscillations known as limit cycles, resulting in a periodic output even after the input signal has been removed. We assume that the quantizers use magnitude truncation in the absence of an overflow, and two's-complement overflow followed by magnitude truncation otherwise. In circuits using such quantizers, two types of limit cycles are commonly observed, namely granular oscillations and overflow oscillations [71]. A sufficient condition [72] for the absence of zero-input limit cycles is the existence of a diagonal matrix $D_{0}$ of positive elements such that ${ }^{4}$

$$
D_{0}-A^{\prime} D_{0} A \geq 0 \text {. }
$$

In view of condition (9.6a), we see that (9.21) is satisified with $D_{0}=I$. That is, the energy balance condition ensures the absence of limit cycles. Moreover, if $\boldsymbol{A}$ satisfies condition (9.21), so does $T^{-1} A T$ for any diagonal transformation matrix $T$, simply by replacing $D_{0}$ with $T D_{0} T$. Hence scaling does not sacrifice the freedom from limit cycle property, and accordingly, all the Gray-Markel lattice structures are devoid of limit cycles [14], [72].

\section{Applications to Complex All-Pass Functions}

In quoting the discrete-time LBR lemma we have assumed real coefficient filtering. The many results above easily generalize to complex filters by replacing, for example, matrix transposition operations with conjugate transposition operations. As such, one can show that complex filters which satisfy the energy balance constraint are minimum noise structures and are free from limit cycles. Thus the complex lattice filters, for example, share the attractive properties of their real arithmetic counterparts.

Complex all-pass filters derived from other methods have desirable finite word-length properties as well. For example, the complex transformation of (3.7) may be interpreted as a diagonal similarity transformation $T$, such that

$$
\boldsymbol{I}=\operatorname{diag}\left\{\mathrm{e}^{i \phi_{k}}\right\} \text {. }
$$

This transformation matrix is unitary, and as such has no effect on the energy balance properties (or lack thereof) of the structure to which it is applied.

Finally, the (nonminimal) first-order complex all-pass structure of Fig. 7 admits the state-space description

$$
\begin{aligned}
{\left[\begin{array}{l}
x_{1}(n+1) \\
x_{2}(n+1)
\end{array}\right]=} & {\left[\begin{array}{ll}
a+j b & -1 \\
0 & 0
\end{array}\right]\left[\begin{array}{l}
x_{1}(n) \\
x_{2}(n)
\end{array}\right] } \\
& +\left[\begin{array}{l}
a-j b \\
1
\end{array}\right] u(n) \\
y(n)= & {[a+j b-1]\left[\begin{array}{l}
x_{1}(n) \\
x_{2}(n)
\end{array}\right]+(a-j b) u(n) }
\end{aligned}
$$

${ }^{4}$ The inequality in (9.21) indicates that the (symmetric) matrix on the left is positive semi-definite. where $a+j b=\gamma$ is the pole of the filter. Upon setting the input $u(n)$ to zero, the feedback portion reduces to

$$
x_{7}(n+1)=\gamma x_{1}(n) .
$$

Now, stability of the filter implies

$$
1-|\gamma|^{2}>0
$$

which, in view of (9.21) (with $D_{0}=1$ and $A=\gamma$ ), ensures the absence of zero-input limit cycles.

\section{Concluding Remarks}

In this paper we have outlined the use of all-pass filters in a variety of signal processing applications, including complementary filtering and filter banks, multirate filtering, frequency response equalization, etc. Fundamental to many of these results is the lossless property exhibited by an all-pass function; provided this property is structurally induced, the desirable features in each application exhibit very robust performance in the face of coefficient quantization. Furthermore, by using filter structures which satisfy the energy balance relation of Section IX, limit cycles are avoided and the roundoff noise of the filter is minimized.

\section{ACKNOWLEDGMENT}

The authors would like to thank Dr. M. Bellanger for useful criticisms.

\section{ReFERENCES}

[1] A. Fettweis, "Pseudopassivity, sensitivity, and stability of wave digital filters," IEEE Trans. Circuits Syst., vol. CT-19, pp. 668673, Nov. 1972.

[2] P. P. Vaidyanathan and S. K. Mitra, "Low passband sensitivity digital filters: A generalized viewpoint and synthesis procedures," Proc. IEEE, vol. 72, pp. 404-423, Apr. 1984.

[3] _- "A general family of multivariable digital lattice filters," IEEE Trans. Circuits Syst., vol. CAS-32, pp. 1234-1245, Dec. 1985.

[4] A. Fettweis, "Multidimensional wave digital filters-Problems and progress," in Proc. 1986 Int. Conf. on Circuits and Systems, (San Jose, CA, May 1986), pp. 506-509.

[5] A. V. Oppenheim and R. W. Schafer, Digital Signal Processing. Englewood Cliffs, NJ: Prentice-Hall, 1975.

[6] S. K. Mitra, P. A. Regalia, and P. P. Vaidyanathan, "Bounded complex transfer function, and its application to low sensitivity filter design," in Proc. 1986 Int. Symp. on Circuits and Systems (San Jose, CA, May 1986), pp. 452-455.

[7] S. K. Mitra and K. Hirano, "Digital allpass filters," IEEE Trans. Circuits Syst., vol. CAS-21, pp. 688-700, Sept. 1974.

[8] J. Szczupak, S. K. Mitra, and J. Fadavi, "Realization of structurally LBR digital allpass filters," in Proc. IEEE Int. Symp. on Circuits and Systems (Philadelphia, PA, May 1987), pp. 633636.

[9] L. B. Jackson, "Digital phase equalizer," U.S. Patent 3537015 , Oct. 1970.

[10] B. Liu and R. Ansari, "A class of low-noise computationally efficient recursive digital filters, with applications to sampling rate alterations," IEEE Trans. Acoust., Speech, Signal Processing, vol. ASSP-33, pp. 90-97, Feb. 1985.

[11] A. H. Gray, Jr., and J. D. Markel, "Digital lattice and ladder filter synthesis," IEEE Trans. Audio Electroacoust., vol. AU-21, pp. 491-500, 1973.

[12] P.P. Vaidyanathan and S. K. Mitra, "A unified structural interpretation of some well-known stability tests for linear systems," Proc. IEEE, vol. 75, pp. 478-497, Apr. 1987.

[13] A. H. Gray, Jr., and J. D. Markel, "A normalized filter structure," IEEE Trans. Acoust., Speech, Signal Processing, vol. ASSP-23, pp. 268-270, 1975.

[14] A. H. Gray, Jr., "Passive cascaded lattice digital filters," IEEE Trans. Circuits Syst., vol. CAS-27, pp. 337-344, May 1980. 
[15] J. E. Volder, "The CORDIC trigonometric computing technique," IRE Trans. Electron. Comput., vol. EC-8, pp. 330-334, Sept. 1959.

[16] P. P. Vaidyanathan, P. A. Regalia, and S. K. Mitra, "Design of doubly complementary IIR digital filters using a single complex allpass filter, with multirate applications," IEEE Trans. Circuits Syst., vol. CAS-34, pp. 378-389, Apr. 1987.

[17] T. Takebe, K. Nishikawa, and M. Yamamoto, "Complex coefficient digital allpass networks and their applications to variable delay equalizer design," in Proc. 1980 lnt. Symp. on Circuits and Systems (Houston, TX, Apr. 1980), pp. 605-608.

[18] A. Fettweis, "Principles of complex wave digital filters," Int. J. Circuit Theory Appl., vol. 9, pp. 119-134, Apr. 1981.

[19] - "Wave digital filters: Theory and practice," Proc. IEEE, vol. 74, pp. 270-327, Feb. 1986

[20] K. Hirano, S. Nishimura, and S. K. Mitra, "Digital notch filters," IEEE Trans. Commun., vol. COM-22, pp. 964-970, July 1974.

[21] T. Saramäki, T.-H. Yu, and S. K. Mitra, "Very low sensitivity realization of IIR digital filters using a cascade of complex allpass structures," IEEE Trans. Circuits Syst., vol. CAS-34, pp. 876-886, Aug. 1987.

[22] P.P. Vaidyanathan, S. K. Mitra, and Y. Neuvo, "A new method of low sensitivity filter realization," IEEE Trans. Acoust., Specch, Signal Processing, vol. ASSP-34, pp. 350-361, Apr. 1986.

[23] A. Fettweis, H. Levin, and A. Sedlmeyer, "Wave digital lattice filters," Int. J. Circuit Theory Appl., vol. 2, pp. 203-211, 1974.

[24] L. Gazsi, "Explicit formulas for lattice wave digital filters," IEEE Trans. Circuits Syst., vol. CAS-32, pp. 68-88, Jan. 1985.

[25] T. Saramäki, "On the design of digital filters as the sum of two all-pass filters," IEEE Trans. Circuits Syst., vol. CAS-32, pp. 1191-1193, Nov. 1985.

[26] P. A. Regalia and S. K. Mitra, "Low sensitivity active filter realization using a complex allpass filter," IEEE Trans. Circuits Syst., vol. CAS-34, pp. 390-399, Apr. 1987.

[27] H. J. Orchard, "Inductorless filters," Electron. Lett., vol. 2, pp. 224-225, Sept. 1966.

[28] L. B. Jackson, Digital Filters and Signal Processing. Boston, MA: Kluwer, 1986.

[29] S. K. Mitra, Y. Neuvo, and P. P. Vaidyanathan, "Complementary IIR digital filter banks," in Proc. IEEE Int. Conf. on Acoustics, Speech, and Signal Processing (Tampa, FL, Mar. 1985), pp. 529-532.

[30] R. Ansari and B. Liu, "Efficient sampling rate alteration using (IIR) digital filters," IEEE Trans. Acoust., Speech, Signal Processing, vol. ASSP-31, pp. 1366-1373, 1983.

[31] W. Drews and L. Gazsi, "A new design method for polyphase filters using all-pass sections," IEEE Trans. Circuits Syst., vol. CAS-33, pp. 346-348, Mar. 1986.

[32] M. Renfors and T. Saramäki, "Recursive $N$-th band digital filters," IEEE Trans. Circuits Syst., vol. CAS-34, pp. 24-51, Jan. 1987.

[33] R. E. Crochiere and L. Rabiner, Multirate Digital Signal Processing. Englewood Cliffs, NJ: Prentice-Hall, 1983.

[34] N. Ahmed and K. Rao, Orthogonal Transforms for Digital Sig. nal Processing. New York, NY: Springer-Verlag, 1975.

[35] D. F. Elliot and K. R. Rao, Fast Transforms. New York, NY: Academic Press, 1982.

[36] T. A. Ramstad and O. Foss, "Subband coder design using recursive quadrature mirror filters," in Signal Processing: Theories and Applications, M. Kunt and F. deCoulon, Eds. Amsterdam, The Netherlands: North-Holland, 1980.

[37] P. C. Millar, "Recursive quadrature mirror filters-Criteria specification and design method," IEEE Trans. Acoust., Speech, Signal Processing, vol. ASSP-33, pp. 413-420, Apr. 1985.

[38] A. Fettweis, J. A. Nossek, and K. Meerkötter, "Reconstruction of signals after filtering and sampling rate reduction," IEEE Trans. Acoust., Speech, Signal Processing, vol. ASSP-33, pp. 893-902, Aug. 1985.

[39] C. R. Galand and H. J. Nussbaumer, "New quadrature mirror filter structures," IEEE Trans. Acoust., Speech, Signal Processing, vol. ASSP-32, pp. 522-531, June 1984.

[40] K. Meerkötter, "Antimetric wave digital filters derived from complex reference circuits," in Proc. 1983 European Conf. on Circuit Theory and Design (Stuttgart, FRG, Sept. 1983), pp. 217220.
[41] C. M. Rader, "A simple method for sampling in-phase and quadrature components," IEEE Trans. Aerosp. Electron. Syst. vol. AES-20, pp. 821-823, Nov. 1984.

[42] P. A. Regalia and S. K. Mitra, "Quadrature mirror Hilbert transformers," to appear in Digital Signal Processing, V. Cappellini and A. G. Constantinidies, Eds. Amsterdam, The Netherlands: North-Holland, 1987.

[43] B. Gold and C. M. Rader, Digital Processing of Signals. New York, NY: McGraw-Hill, 1969, pp. 90-92.

[44] K. Meerkötter and M. Romeike, "Wave digital Hilbert transformers," in Proc. IEEE Int. Symp. on Circuits and Systems (Montreal, P.Q., Canada, 1984), pp. 258-260.

[45] H. W. Schüssler and J. Weith, "On the design of recursive Hilbert transformers," in Proc. Int. Conf. on Acoustics, Speech, Signal Processing (Dallas, TX, Apr. 1987), pp. 876-879.

[46] R. Ansari, "IIR discrete-time Hilbert transformers," IEEE Trans. Acoust., Speech, Signal Processing, vol. ASSP-35, pp. 11161119, Aug. 1987.

[47] L. B. Jackson, "On the relationship between digital Hilbert transformers and certain lowpass filters," IEEE Trans. Acoust., Speech, Signal Processing, vol. ASSP-23, pp. 381-383, Aug. 1975.

[48] A. G. Constantinidies, "Spectral transformations for digital filters," Proc. Inst. Elec. Eng., vol. 117, pp. 1585-1590, Aug. 1970.

[49] P. A. Regalia and S. K. Mitra, "Tunable digital frequency response equalization filters," IEEE Trans. Acoust., Speech Signal Processing, vol. ASSP-35, pp. 118-120, Jan. 1987.

[50] E. H. J. Persoon and C. J. B. Vandenbulcke, "Digital audio: Examples of the application of the ASP integrated signal processor," Philips Tech. Rev., vol. 42, pp. 201-216, Apr. 1986.

[51] J. K. J. Van Ginderduen et al., "A high quality digital audio filter set designed by silicon compiler CATHEDRAL-I," IEEE J. Solid-State Circuit, vol. SC-21, pp. 1067-1075, Dec. 1986.

[52] J. A. Moorer, "The manifold joys of conformal mapping: Applications to digital filtering in the studio," J. Audio Eng. Soc., vol. 31, pp. 826-841, Nov. 1983.

[53] S. K. Mitra, Y. Neuvo, and H. Roivainen, "Variable cutoff frequency digital filters," in Proc. IASTED Int. Symp. on Applied Signal Processing and Digital Filtering (Paris, France, June 1985), pp. 5-8.

[54] R. Ansari, "Multilevel IIR digital filters," IEEE Trans. Circuits Syst., vol. CAS-32, pp. 337-341, Nov. 1985.

[55] C. W. Kim and R. Ansari, "Piecewise-constant magnitude filters using allpass sections," Electron. Lett., vol. 22, pp. 10071008, Sept. 1986.

[56] A. G. Deczky, "General expression for the group delay of digital filters," Electron. Lett., vol. 5, pp. 663-665, Dec. 11, 1969.

[57] E. W. Cheney, Introduction to Approximation Theory. New York, NY: McGraw-Hill, 1966.

[58] A. G. Deczky, "Equiripple and minimax (Chebyshev) approximations for recursive digital filters," IEEE Trans. Acoust., Speech, Signal Processing, vol. ASSP-22, pp. 98-111, Apr. 1974.

[59] _- "Synthesis of recursive digital filters using the minimum p-error criterion," IEEE Trans. Audio Electroacoust., vol. AU20, pp. 257-263, Oct. 1972.

[60] J.-P. Thiran, "Equal-ripple delay recursive digital filters," IEEE Trans. Circuit Theory, vol. CT-18, pp. 664-669, Nov. 1971.

[61] A. G. Deczky, "Recursive digital filters having equiripple group delay," IEEE Trans. Circuits Syst., vol. CAS-31, pp. 131134, Jan. 1974.

[62] T. Saramäki and Y. Neuvo, "Digital filters with equiripple magnitude and group delay," IEEE Trans. Acoust., Speech, Signal Processing, vol. ASSP-32, pp. 1194-1200, Dec. 1984.

[63] C.W. Kim and R. Ansari, "Approximately linear phase IIR filters using allpass sections," in Proc. 1986 Int. Symp. on Circuits and Systems (San Jose, CA, May 1986), pp. 661-664.

[64] $M$. Refors and T. Saramäki, "A class of approximately linear phase digital filters composed of allpass subfilters," in Proc. 1986 int. Symp. on Circuits and Systems (San Jose, CA, May 1986), pp. 678-681.

[65] P. P. Vaidyanathan, "The discrete-time bounded-real lemma in digital filtering," IEEE Trans. Circuits Syst., vol. CAS-32, pp. 918-924, Sept. 1985.

[66] C.V. K. Prabhakara Rao and P. Dewilde, "On lossless transfer functions and orthogonal realizations," IEEE Trans. Circuits Syst., vol. CAS-34, pp. 677-678, June 1987.

[67] E. DeWilde and P. Deprettere, "Orthogonal cascade real- 
ization of real multiport digital filters," Int. J. Circuit Theory Appl., vol. 8, pp. 245-277, 1980.

[68] S. K. Rao and T. Kailath, "Orthogonal digital filters for VLSI implementation," IFEE Trans. Circuits Syst., vol. CAS-31, pp. 933-945, Nov. 1984

[69] C. T. Mullis and R. A. Roberts, "Synthesis of minimum roundoff noise fixed point digital filters," IEEE Trans. Circuits Syst., vol. CAS-23, pp. 551-562, Sept. 1976.

[70] B. D. O. Anderson and J. Moore, Optimal Filtering. Englewood Cliffs, NJ: Prentice-Hall, 1979.

[71] T. A. C. M. Claasen, W. F. G. Mecklenbrauker, and J. B. H. Peek, "Effects of quantization and overflow in recursive digital filters," IEEE Trans. Acoust., Speech, Signal Processing, vol. ASSP-24, pp. 517-529, Dec. 1976.

[72] P. P. Vaidyanathan and V. Liu, "An improved sufficient condition for the absence of limit cycles in digital filters," IFEF Trans. Circuits Syst., vol. CAS-34, pp. 319-322, Mar. 1987.

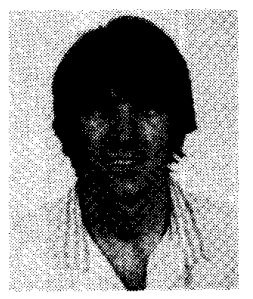

Phillip A. Regalia (Student Member, IEEE) was born in Walnut Creek, CA, in 1962. He received the $\mathrm{B} . \mathrm{Sc}$. degree (highest honors) in electrical engineering from the University of California at Santa Barbara in 1985. Presently, he is a Ph.D. candidate at UCSB, where he works as a research assistant in the Signal Processing Laboratory. His research interests include analog circuit theory and digital signal processing.

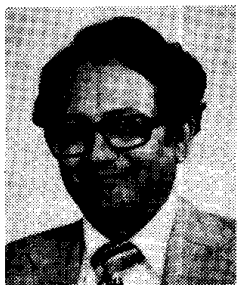

Sanjit K. Mitra (Fellow, IEEE) received the B.Sc. (Hons.) degree in physics in 1953 from Utkal University, Cuttack, India; the M.Sc. (Tech.) degree in radio physics and electronics in 1956 from Calcutta University, Calcutta, India; and the M.S. and Ph.D. degrees in electrical engineering from the University of California, Berkeley, in 1960 and 1962, respectively. In May 1987, he was awarded an Honorary Doctorate of Tech nology degree by the Tampere University of Technology, Tampere, Finland.
He was a member of the faculty of the Cornell University, Ithaca, NY, from 1962 to 1965 , and a member of the Technical Staff of the Bell Laboratories from 1965 to 1967 . He joined the faculty of the University of California, Davis, in 1967, and transferred to the Santa Barbara campus in 1977 as a Professor of Electrical and Computer Engineering, where he served as Chairman of the Department from July 1979 to June 1982. He has held visiting appointments at universities in Australia, Brazil, Finland, India, West Germany, and Yugoslavia. He is the recipient of the 1973 F.E. Terman Award and the 1985 AT\&T Foundation Award of the American Society of Engineering Education, a Visiting Professorship from the Japan Society for Promotion of Science in 1972, and the Distinguished Fulbright Lecturer Award for Brazil in 1984 and Yugoslavia in 1986.

Dr. Mitra is a Fellow of the AAAS, and a member of the ASEE, EURASIP, Sigma Xi, and Eta Kappa Nu. He is a member of the Advisory Council of the George R. Brown School of Engineering of the Rice University, Houston, TX and an Honorary Professor of the Northern Jiaotong University, Beijing, China.

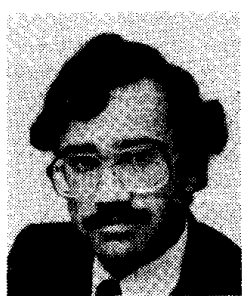

P. P. Vaidyanathan (Member, IEEE) was born in Calcutta, India, on October 16, 1954. He received the B.Sc. (Hons.) degree in physics, and the B.Tech. and M.Tech. degrees in radiophysics and electronics from the University of Calcutta, India, in 1974, 1977, and 1979, respectively, and the Ph.D. degree in electrical and computer engineering from the University of California, Santa Barbara, in 1982.

He was a Postdoctoral Fellow at the University of California, Santa Barbara, from September 1982 to February 1983. Since March 1983 he has been with the California Institute of Technology, Pasadena, as an Assistant Professor of Electrical Engineering. His main research interests are in digital signal processing, linear systems, and filter design. He was the recipient of the Award for Excellence in Teaching at the California Institute of Technology for 1983-1984, and a recipient of NSF's Presidential Young Investigator Award, starting from the year 1986.

Dr. Vaidyanathan served as the Vice Chairman of the Technical Program Committee for the 1983 IEEE International Symposium on Circuits and Systems. He currently serves as an Associate Editor of the IEEE TRANSACTIONS ON CiRCUITS AND SYSTEMS. 